\title{
Geoquímica de las rocas metasedimentarias del Cámbrico medio al Ordovícico temprano de la Sierra de Los Llanos (Sierras Pampeanas, Argentina): Fuente de sedimentos, correlación y ambiente geotectónico
}

\author{
*Sebastián O. Verdecchia ${ }^{1}$, Juan A. Murra ${ }^{1}$, Edgardo G. Baldo ${ }^{1}$, Cesar Casquet ${ }^{2}$, \\ Inmaculada Pascua ${ }^{3}$, Julio Saavedra ${ }^{4}$
}

\author{
${ }^{1}$ CICTERRA-CONICET-UNC, Facultad de Ciencias Exactas, Físicas y Naturales, Universidad Nacional de Córdoba, Avda. Vélez \\ Sarsfield 1611, C.P. X5016CGA-Córdoba, Argentina. \\ sverdecchia@gmail.com; jmurra@com.uncoredu; ebaldo@com.uncor.edu \\ ${ }^{2}$ Departamento de Petrología y Geoquímica, Facultad de Ciencias Geológicas e Instituto de Geociencias IGEO (Universidad Com- \\ plutense, CSIC), 28040 Madrid, España. \\ casquet@geo.ucm.es \\ ${ }^{3}$ Investigación y Control de Calidad, S.A.-INCOSA, Parque Tecnológico de Boecillo, Recinto 2. 47151 Boecillo, Valladolid, España. \\ mipascuag@incosa.es \\ ${ }^{4}$ Instituto de Agrobiología y Recursos Naturales CSIC, 37071 Salamanca, España. \\ julio.saavedra@irnasa.csic.es
}

* Correspondencia autor: sverdecchia@gmail.com

\begin{abstract}
RESUMEN. El complejo metamórfico de la Sierra de Los Llanos está constituido por rocas paraderivadas metamorfizadas en condiciones de bajo a alto grado y representadas por filitas, esquistos, gneises y migmatitas que afloran como grandes septos dentro de las unidades magmáticas del Ordovícico inferior. Localmente se reconocen rocas corneanas asociadas a la intrusión de cuerpos de composición básica. Las rocas metasedimentarias de este complejo muestran composiciones químicas equivalentes a pelitas y areniscas del tipo sublitoarenita y subarcosa. Las relaciones de Ti/Zr $(5,4-8,4), \mathrm{La} / \mathrm{Sc}(5,2-8,5), \mathrm{Th} / \mathrm{Sc}>1(2,2-3,4), \mathrm{La} / \mathrm{Th}(2,0-2,5)$ y $\mathrm{Zr} / \mathrm{Sc}(60-103)$, junto a las concentraciones de $\mathrm{Hf}(7,9$ a $12,4 \mathrm{ppm})$ y Sc $(4,4-5,2 \mathrm{ppm})$ en las metapsamitas son indicativas de una fuente enriquecida en componentes félsicos o de materiales reciclados y con reducida participación de componentes máficos. La correlación geoquímica de estas unidades con las metapsamitas de los complejos metamórficos Chepes, La Cébila y Pringles sugiere una misma composición del área fuente para todos ellos. Las concentraciones de Ti, Zr, Hf, La, Th y Sc son variables y no permiten definir claramente un determinado contexto geotectónico, sugiriendo además que la composición de los protolitos fue mayormente controlada por procesos sedimentarios y no por la heterogeneidad composicional de las fuentes. La integración de la información geoquímica con los datos geocronológicos conocidos y la correlación regional de los complejos metamórficos ordovícicos permite reforzar la propuesta de definir como principales áreas fuente de estos complejos las rocas ígneas y metamórficas de la faja orogénica Pampeana y del Cratón del Río de La Plata. La información aportada es consistente, además, con la existencia de una extensa plataforma continental en el margen suroccidental de Gondwana durante el Cámbrico medio al Ordovícico temprano.
\end{abstract}




\begin{abstract}
Geochemistry of middle Cambrian to early Ordovician metasedimentary rocks of the Sierra de Los Llanos (Sierras Pampeanas, Argentina): Source of sediments, correlations and geotectonic setting. The metamorphic complex of Sierra de Los Llanos is composed of low- to high-grade metasedimentary rocks, namely phyllites, schists, gneisses and migmatites that out crop like large septa within lower Ordovician magmatic units. Cornubianite associated with the intrusion of basic composition bodies are locally recognized. Chemical compositions of these metasedimentary rocks are equivalent to pelite and arenite (sublitharenite-subarkose). Chemical ratios of $\mathrm{Ti} / \mathrm{Zr}$ (5.4-8.4), $\mathrm{La} / \mathrm{Sc}$ (5.2-8.5), Th/Sc $>1$ (2.2-3.4), La/Th (2.0-2.5) and Zr/Sc (60-103), along with contents of Hf (7.9 a 12.4 ppm) and $\mathrm{Sc}(4.4-5.2 \mathrm{ppm})$ in meta-psammites, suggest that the source of the sediments was enriched in felsic components or recycled material and limited participation mafic components. A similar composition of source areas for meta-psammites of this complex and Chepes, La Cébila and Pringles metamorphic complexes is suggested from geochemical data. Ti, $\mathrm{Zr}$, Hf, La, Th and Sc concentrations are variable and do not allow to clearly define a specific geotectonic context and suggest that the composition of the protoliths was largely controlled by sedimentary processes rather than compositional heterogeneity of the sources. The integration of geochemical and known geochronological data and the regional correlation of Ordovician metamorphic complexes strengthen the interpretation that defines igneous and metamorphic rocks from Pampean orogenic belt and Río de La Plata craton as the mayor sources of these complexes. Furthermore, this information is consistent with the existence of an extensive continental shelf in the southwestern margin of Gondwana during the Middle Cambrian to Early Ordovician.
\end{abstract}

Keywords: Los Llanos metamorphic complex, Geochemistry of metasedimentary rocks, Late Cambrian, Pampean orogeny, Famatinian orogeny, Eastern Sierras Pampeanas.

\section{Introducción}

La composición química de las rocas sedimentarias y metasedimentarias de bajo a medio grado permite definir las características geoquímicas del área fuente $y$, por extensión, determinar el ambiente geotectónico de las cuencas sedimentarias (Bhatia y Crook, 1986; McLennan et al., 1990, 2003). Durante los procesos de meteorización, diagénesis y metamorfismo de bajo grado, algunos elementos mayores y trazas son susceptibles de movilizarse y migrar del sistema, especialmente debido a la presencia de fluidos (e.g., $\mathrm{Si}, \mathrm{Al}, \mathrm{Fe}, \mathrm{Mg}, \mathrm{Mn}, \mathrm{Ca}, \mathrm{K}$, $\mathrm{Ba}, \mathrm{Sr}, \mathrm{Rb}$ y $\mathrm{Cs}$ ). Por lo tanto, estos elementos no son adecuados para discriminar ambientes geotectónicos y caracterizar el área fuente. Por otra parte, algunos elementos mayores (e.g., P y Ti) y trazas (e.g., La, Ce, Nd, Y, Th, Zr, Hf, Nb y Sc), que están contenidos en minerales detríticos resistentes a la alteración o que no se fraccionan durante los procesos de alteración-transporte-sedimentación, conservan las relaciones interelementales iniciales siendo más útiles para ese fin (Bhatia y Crook, 1986; Floyd y Leveridge, 1987; McLennan, 1989; McLennan et al., 1990; Armstrong-Altrina y Vermaa, 2005).

Las Sierras Pampeanas Orientales (centro-oeste de Argentina, figura 1a) constituyen una serie de alineaciones montañosas de dirección N-S en las que afloran complejos ígneos y metamórficos vinculados a la historia temprana del extremo suroccidental (coordenadas actuales) de Gondwana, abarcando desde el Neoproterozoico al Paleozoico medio a tardío (Pankhurst y Rapela, 1998; Steenken, et al., 2011). Las dos orogénesis mejor conocidas, y que afloran más extensamente dentro de esta región, son la Pampeana (Neoproterozoico superior-Cámbrico inferior; ca. 550-515 Ma) y la Famatiniana (Cámbrico tardío a Ordovícico; $c a$. 500-440 Ma), a las que siguen eventos más discretos de magmatismo y deformación devónico-carboníferos asociados a la orogenia Achaliana (e.g., Rapela et al., 1998; Sims et al., 1998; Pankhurst et al., 1998, 2000; Dahlquist et al., 2010; Otamendi et al., 2008, 2012; Rapela et al., 2007; Siegesmund et al., 2010; Steenken, et al., 2011; Casquet et al., 2012). En la última década se ha incrementado significativamente la información geoquímica, isotópica y geocronológica (especialmente $\mathrm{U}-\mathrm{Pb}$ en circones detríticos) de las rocas metasedimentarias de la región de las Sierras Pampeanas Orientales (e.g., Sims et al., 1998; López de Luchi et al, 2003; Escayola et al., 2007; Rapela et al., 2007; Collo et al., 2009; Drobe et al., 2009, 2011; Verdecchia y Baldo, 2010; Adams et al., 2011; Verdecchia et al., 2011; Murra et al., 2011; Larrovere et al., 2012; Iannizzotto et al., 2013), permitiendo discriminar las diferentes paleocuencas, principalmente aquellas que funcionaron durante el Neoproterozoico tardío al Ordovícico temprano.

El objetivo de este trabajo es caracterizar geoquímicamente las rocas metasedimentarias del Complejo 
Metamórfico Los Llanos ubicado en la Sierra de Los Llanos-Chepes-Ulapes, dentro de la región de las Sierras Pampeanas Orientales (Fig. 1). En esta sierra, el metamorfismo y el plutonismo han sido vinculados con la orogenia Famatinana (Pascua et al., 1998; Pankhurst et al., 1998, 2000). Los nuevos datos químicos de elementos mayores y trazas permiten definir las características composicionales de las áreas fuente y, además, la combinación de esta información con las edades publicadas del metamorfismo y magmatismo permiten acotar la edad de sedimentación de las rocas paraderivadas de la
Sierra de Los Llanos y correlacionarlas con otras sucesiones metasedimentarias de la misma edad en el ámbito de las Sierras Pampeanas.

\section{Marco geológico}

La Sierra de Los Llanos se localiza en el sector septentrional del cordón serrano Los Llanos-ChepesUlapes (Sierras Pampeanas Orientales, provincia de La Rioja, figura 1a y b). Estas sierras están constituidas por cuerpos plutónicos de composición calcoalcalina, dominantemente metaluminosos, con-

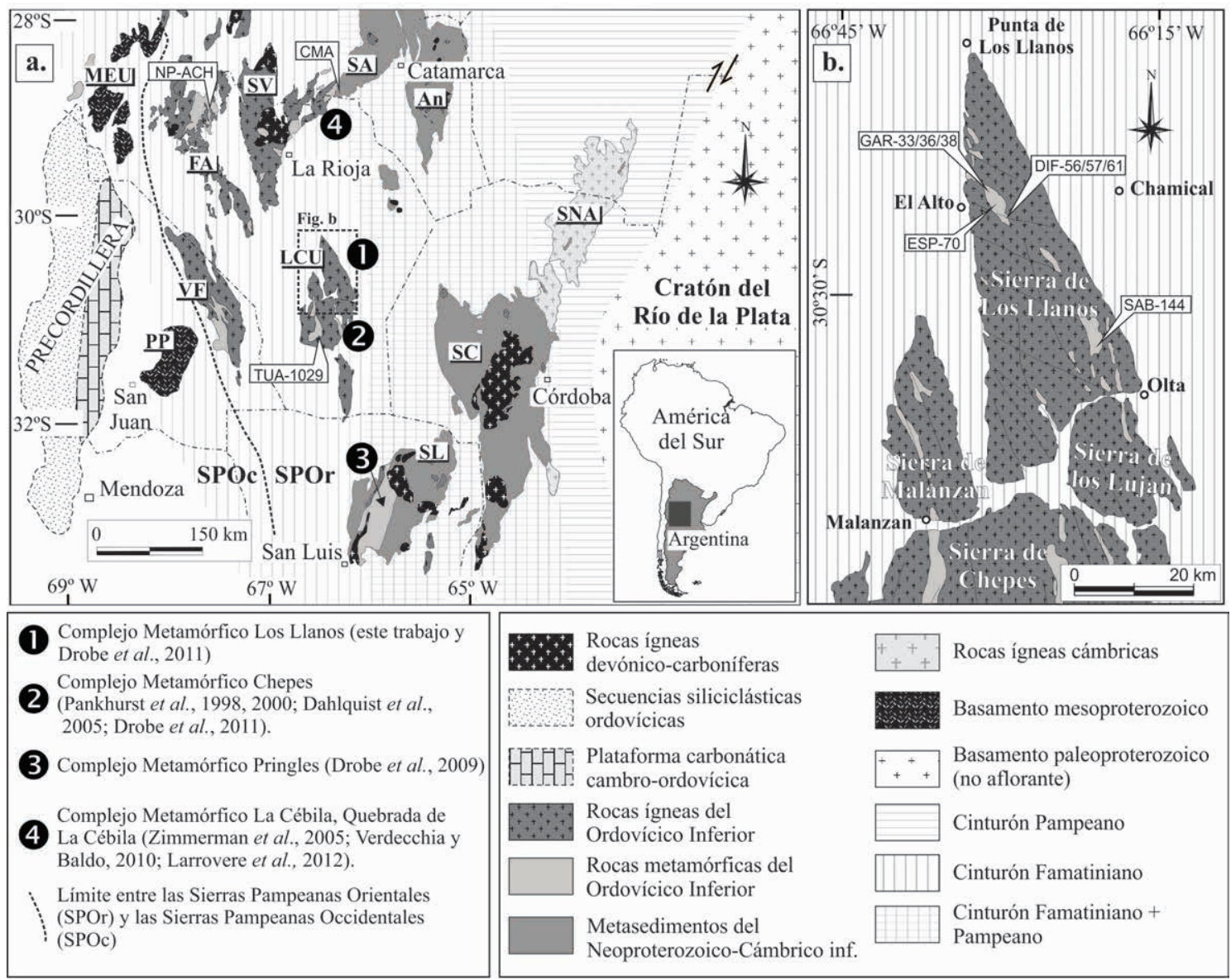

FIG. 1. a. Mapa geológico sintético de las Sierras Pampeanas (centro de Argentina), que muestra las principales sierras y cinturones orogénicos junto a los complejos metamórficos estudiados en este trabajo (modificado de Steenken et al., 2006 y Verdecchia et al., 2011). Se indica la ubicación de las muestra de granito TUA-1029 de Pankhurst et al. (2000); b. Detalle de la Sierra de Los Llanos y el sector norte de la Sierra de Chepes y colindantes, que señala los puntos de muestreo. Sierra de Córdoba (SC), Sierra Norte-Ambargasta (SNA), Sierra de San Luis (SL), Sierras de Los Llanos-Chepes-Ulapes (LCU), Sierra de Ancasti (An), Sierra de Ambato (SA), Sierra de Velasco (SV), Sierra de Famatina (FA), Sierra de Valle Fértil (VF), Sierra de Pie de Palo (PP), Sierras de Maz-Espinal-Umango (MEU), Complejo Metamórfico Ambato (CMA), Formaciones Negro Peinado y Achavil (NP-ACH). Se señala la ubicación de la muestra de metapsamita GAR-33 publicada por Dahlquist et al. (2005). 
siderados como la extensión norte del batolito Los Llanos-Ulapes (Pankhurst et al., 1998 y referencias allí citadas). La litología ígnea dominante es de composición granodiorítica y contiene abundantes enclaves microgranulares máficos de composición tonalítica. La edad de cristalización de la unidad granodiorítica muestra un rango de edades de 483497 Ma (Pankhurst et al., 1998, 2000; Dahalquist et al., 2005). En menor proporción se reconocen monzogranitos intruyendo a la granodiorita (Unidad Asperezas, de ca. $477 \mathrm{Ma}$; Pankhurst et al., 2000), gabros, dioritas en relación de mezcla con la granodiorita y finalmente granitos anatécticos como el Granito Tuani (ca. $479 \mathrm{Ma}$; Pankhurst et al., 2000, Dahlquist et al., 2005). El magmatismo de esta sierra, tanto metaluminoso como peraluminoso, registra valores negativos de $\varepsilon \mathrm{Nd}(-4,2$ a $-6,7)$ que sugieren una componente isotópica continental en la fuente (Pankhurst et al., 1998).

Las rocas metasedimentarias afloran de manera discontinua e intercalada con las unidades plutónicas, constituyendo grandes septos de dimensiones kilométricas. Estos septos representan fragmentos de una sucesión sedimentaria psamítico-pelítica que fue metamorfizada en condiciones de baja presión y con desarrollo de una zonación de bajo a alto grado (Pascua et al., 1998; Pankhurst et al., 1998). Limarino y Poma (1999) han agrupado las rocas metamórficas de la Sierra de Los Llanos en dos unidades formacionales en función del grado metamórfico: la Formación Olta (que incluye a filitas, pizarras, metapsamitas, esquistos y cuarcitas) y el Complejo Pacatala (que agrupa a gneises y migmatitas). En este trabajo se prefiere utilizar la denominación de Complejo Metamórfico Los Llanos (CMLL) para referirnos a todas las unidades metamórficas del área por considerar que las diferencias en grados metamórficos no son un criterio válido para diferenciar unidades formacionales. Junto a las litologías mencionadas, se han reconocido también niveles subordinados de rocas calcosilicáticas y corneanas localizadas en las cercanías de los intrusivos más básicos (Pascua et al., 1998).

Las rocas plutónicas y metamórficas de la Sierra de Los Llanos, Chepes y Ulapes constituyeron el basamento cristalino sobre el que se depositaron los sedimentos continentales del grupo Paganzo (Carbonífero-Pérmico). La tectónica compresiva de la orogenia Andina (Mioceno-Plioceno) facilitó la exhumación a nivel de erosión de este basamento, debido al basculamiento de grandes bloques corticales a favor de fallas inversas de alto ángulo y buzamiento al oeste (Limarino y Poma, 1999).

\section{Muestras y metodología}

Con el objeto de determinar la señal geoquímica de las unidades metasedimentarias del CMLL, se analizaron siete muestras del CMLL: dos muestras que corresponden a metapsamitas (Gar-38 y Dif-56) y cinco a metapelitas (Sab-144, Dif-61, Gar-36, Dif-57 y Esp70 ), provenientes de las unidades de bajo y alto grado y cuya ubicación puede observarse en la figura 1b). Por otro lado, en el tratamiento de la información química se incorpora una muestra de esquisto cuarzo-micáceo (metapsamita, Gar-33) publicada por Dahlquist et al. (2005) y, con el objeto de poder comparar la composición de estas muestras con las del Complejo Metamórfico La Cébila (CMLC), se incorporaron nuevos datos de elementos trazas de metapsamitas de la quebrada de La Cébila y la quebrada de Cantadero, incrementando la base de datos de esta región publicadas previamente por Verdecchia y Baldo (2010). Todos los análisis químicos se presentan en el apéndice, y de manera sintética en la tabla 1.

Los análisis químicos de las muestras del CMLL fueron obtenidos siguiendo la rutina (4 Litho-research) de Actlabs (Canadá). Por otra parte, los análisis químicos adicionales de trazas (e.g., REE y Sc) de las muestras del CMLC, fueron completados en el GeoAnalytical Laboratory (Universidad del Estado de Washington, Pullman, Estados Unidos). En ambos laboratorios se aplicó el método de espectrometría de masas con plasma de acoplamiento inductivo (ICP-MS).

\section{Las unidades metasedimentarias del complejo metamórfico Los Llanos}

\subsection{Caracterización geológica y petrográfica de las muestras}

Las rocas metasedimentarias del CMLL afloran como grandes septos, sin una distribución geográfica preferencial. Los de mayor tamaño se encuentran ubicados en la zona noroeste, en las cercanías del paraje El Alto, y en el sector sur, entre las localidades de Olta y Malanzán (Fig. 1b). En uno de los septos de la zona noroeste es donde se observa mejor conservada la secuencia metasedimentaria asociada al 
TABLA 1. PRINCIPALES RELACIONES ENTRE ELEMENTOS QUÍMICOS DE LAS MUESTRAS DEL COMPLEJO METAMÓRFICO LOS LLANOS Y EL COMPLEJO METAMÓRFICO LA CÉBILA. ADEMÁS, SE MUESTRA EL CONTENIDO DE HF DE LAS MUESTRAS ANALIZADAS.

\begin{tabular}{|c|c|c|c|c|c|c|c|c|c|c|c|c|c|c|c|}
\hline \multirow[b]{3}{*}{ Muestra } & \multicolumn{8}{|c|}{ Complejo Metamórfico Los Llanos } & \multicolumn{7}{|c|}{ Complejo Metamórfico La Cébila } \\
\hline & \multicolumn{3}{|c|}{ Metapsamitas } & \multicolumn{5}{|c|}{ Metapelitas } & \multicolumn{7}{|c|}{ Metapsamitas } \\
\hline & $\begin{array}{c}\text { GAR- } \\
\mathbf{3 3}^{1}\end{array}$ & $\begin{array}{c}\text { GAR- } \\
38\end{array}$ & $\begin{array}{c}\text { DIF- } \\
56\end{array}$ & $\begin{array}{c}\text { GAR- } \\
36\end{array}$ & $\begin{array}{c}\text { DIF- } \\
\mathbf{5 7}\end{array}$ & $\begin{array}{c}\text { DIF- } \\
61\end{array}$ & $\begin{array}{c}\text { ESP- } \\
70\end{array}$ & $\begin{array}{c}\text { SAB- } \\
144\end{array}$ & $\begin{array}{l}\text { CAN- } \\
304^{2}\end{array}$ & $\begin{array}{l}\text { CAN- } \\
306^{2}\end{array}$ & $\begin{array}{l}\text { CAN- } \\
318^{2}\end{array}$ & $\begin{array}{l}\text { CEB- } \\
3553^{2}\end{array}$ & $\begin{array}{l}\text { CEB- } \\
354^{2}\end{array}$ & $\begin{array}{l}\text { CEB- } \\
386^{2}\end{array}$ & $\begin{array}{l}\text { CEB- } \\
388^{2}\end{array}$ \\
\hline $\mathrm{Sc} /(\mathrm{v}+\mathrm{Th}+\mathrm{Zr} / 10)$ & 0,1 & 0,07 & 0,11 & 0,33 & 0,33 & 0,36 & 0,36 & 0,32 & 0,05 & 0,06 & 0,09 & 0,24 & 0,23 & 0,18 & 0,22 \\
\hline $\mathrm{Th} /(\mathrm{Sc}+\mathrm{Th}+\mathrm{Zr} / 10)$ & 0,31 & 0,23 & 0,24 & 0,38 & 0,37 & 0,38 & 0,22 & 0,37 & 0,2 & 0,14 & 0,19 & 0,18 & 0,28 & 0,42 & 0,25 \\
\hline $\begin{array}{l}(\mathrm{Zr} / 10) / \\
(\mathrm{Sc}+\mathrm{Th}+\mathrm{Zr} / 10)\end{array}$ & 0,6 & 0,7 & 0,66 & 0,29 & 0,3 & 0,26 & 0,42 & 0,31 & 0,75 & 0,8 & 0,72 & 0,57 & 0,48 & 0,4 & 0,53 \\
\hline $\mathrm{Th} /(\mathrm{Th}+\mathrm{La}+\mathrm{Sc})$ & 0,3 & 0,26 & 0,26 & 0,22 & 0,21 & 0,22 & 0,12 & 0,29 & 0,17 & 0,24 & 0,22 & 0,21 & 0,22 & 0,24 & 0,22 \\
\hline $\mathrm{La} /(\mathrm{Th}+\mathrm{La}+\mathrm{Sc})$ & 0,6 & 0,66 & 0,62 & 0,59 & 0,59 & 0,57 & 0,68 & 0,46 & 0,78 & 0,65 & 0,67 & 0,51 & 0,6 & 0,66 & 0,59 \\
\hline $\mathrm{Sc} /(\mathrm{Th}+\mathrm{La}+\mathrm{Sc})$ & 0,1 & 0,08 & 0,12 & 0,19 & 0,19 & 0,21 & 0,2 & 0,25 & 0,05 & 0,11 & 0,1 & 0,28 & 0,18 & 0,1 & 0,19 \\
\hline $\mathrm{La} / \mathrm{Th}$ & 2 & 2,5 & 2,3 & 2,7 & 2,8 & 2,6 & 5,7 & 1,6 & 4,5 & 2,8 & 3 & 2,4 & 2,8 & 2,7 & 2,7 \\
\hline $\mathrm{Ti} / \mathrm{Zr}$ & 7,2 & 5,4 & 8,4 & 32,7 & 32,9 & 35,8 & 28,9 & 30,2 & 2,4 & 2 & 3,2 & 4,9 & 7,4 & 3,9 & 6,2 \\
\hline $\mathrm{La} / \mathrm{Sc}$ & 6,2 & 8,5 & 5,2 & 3,1 & 3,1 & 2,8 & 3,4 & 1,9 & 16,4 & 6,1 & 6,6 & 1,9 & 3,4 & 6,6 & 3,1 \\
\hline $\mathrm{Th} / \mathrm{Sc}$ & 3,1 & 3,4 & 2,2 & 1,1 & 1,1 & 1,1 & 0,6 & 1,2 & 3,6 & 2,2 & 2,2 & 0,8 & 1,2 & 2,4 & 1,2 \\
\hline $\mathrm{Zr} / \mathrm{Sc}$ & 60,2 & 102,9 & 61,5 & 8,9 & 9,1 & 7,2 & 11,5 & 9,9 & 136,8 & 130,8 & 80,5 & 23,6 & 20,9 & 22,6 & 24,5 \\
\hline $\mathrm{Hf}$ & 7,9 & 12,4 & 9,8 & 5,4 & 6,1 & 4,4 & 5,4 & 5,3 & 7,9 & 11 & 10,7 & 4,9 & 6 & 3,5 & 7,4 \\
\hline $\mathrm{Al}_{2} \mathrm{O}_{3} / \mathrm{K}_{2} \mathrm{O}$ & 2,86 & 5,1 & 6,12 & 4,1 & 4,07 & 3,76 & 3,97 & 3,92 & 15,32 & 15,33 & 29,52 & 6,23 & 3,72 & 7,95 & 4,26 \\
\hline $\mathrm{Rb} / \mathrm{Sr}$ & 1,48 & 1,1 & 0,38 & 3,49 & 1,33 & 3,77 & 2,55 & 2,9 & 0,1 & 0,06 & 0,03 & 14,89 & 0,48 & 0,49 & 1,31 \\
\hline $\mathrm{Eu} / \mathrm{Eu}^{*}$ & 0,62 & 0,56 & 0,61 & 0,59 & 0,7 & 0,61 & 0,64 & 0,67 & 0,72 & 0,67 & 0,62 & 0,22 & 0,68 & 0,71 & 0,6 \\
\hline $\mathrm{Mg} \#: \mathrm{Mg} /(\mathrm{Mg}+\mathrm{Fe})^{3}$ & 0,35 & 0,35 & 0,42 & 0,45 & 0,44 & 0,42 & 0,44 & 0,41 & 0,19 & 0,41 & 0,41 & 0,29 & 0,25 & 0,45 & 0,44 \\
\hline $\mathrm{Cr} / \mathrm{Th}$ & 4,5 & 2,1 & 5,0 & 3,8 & 4,3 & 4,6 & 7,7 & 4,1 & - & - & - & - & - & - & - \\
\hline $\mathrm{V} / \mathrm{Ni}$ & 9,0 & 4,0 & 4,0 & 4,5 & 3,7 & 2,7 & 2,1 & 2,6 & - & - & - & - & - & - & - \\
\hline $\mathrm{CIA}^{4}$ & 59 & 60 & 56 & 76 & 69 & 74 & 72 & 72 & - & - & - & - & - & - & - \\
\hline
\end{tabular}

${ }^{1}$ Muestra extraída de Dahlquist et al. (2005); ${ }^{2}$ Valores de $\mathrm{Al}_{2} \mathrm{O}_{3}, \mathrm{~K}_{2} \mathrm{O}$, La, Th y Hf del Complejo Metamórfico La Cébila fueron publicados en

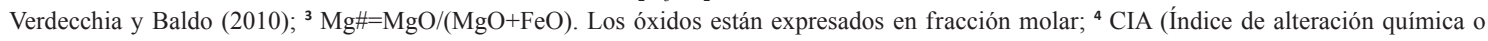
Chemical Index of Alteration $)=\mathrm{Al}_{2} \mathrm{O}_{3} /\left(\mathrm{Al}_{2} \mathrm{O}_{3}+\mathrm{CaO} *+\mathrm{Na}_{2} \mathrm{O}+\mathrm{K}_{2} \mathrm{O}\right.$. El CaO* representa únicamente el perteneciente a los silicatos y corregido según la sugerencia de McLennan (1993).

metamorfismo regional, formada de oeste a este por esquistos andalucíticos y gneises con biotita, sillimanita y cordierita, con intercalaciones esporádicas de niveles centimétricos de rocas calcosilicáticas. En el sector más oriental de este septo, se encuentran migmatitas estromatíticas y diatexitas, mientras que en el sector occidental afloran esquistos moteados (cordierita) y cornubianitas de grano muy fino vinculadas con la intrusión de cuerpos máficos. Las rocas metamórficas presentan una foliación penetrativa de $\sim 310^{\circ} / 75^{\circ} \mathrm{SW}$ dada por la orientación de filosilicatos. En los septos del sector sur, entre Olta y Malanzán
(Fig. 1b), las litologías predominantes son filitas de colores verdosos, intercalados con niveles de metapsamitas de bajo grado. En las proximidades de los cuerpos ígneos, las filitas desarrollan motas no orientadas de biotita.

Filitas (Sab-144, Apéndice, figura 2a). Rocas de color verde y grano muy fino, con textura lepidoblástica con bandas micáceas que envuelven a sectores lentiformes compuestos de cuarzo, plagioclasa y mica. La composición mineralógica es muscovita, clorita, biotita, cuarzo, plagioclasa y minerales accesorios (hematita, circón, apatita). 
Rocas córnenas (Gar-38 y Dif-61, Apéndice, figura $2 b$ ). Se localizan en la región noroccidental de la Sierra de Los Llanos, en contacto con intrusivos de composición máfica (gabros). Constituyen afloramientos continuos de unos $20 \mathrm{~m}$ de potencia. Son de color gris oscuro a negro, de aspecto isótropo y de grano muy fino $(<0,5 \mathrm{~mm})$, con textura granoblástica. La mineralogía varía de cuarzosa a cuarzo-micácea, y se compone de cuarzo, feldespato potásico, plagioclasa, biotita, muscovita, andalucita y cordierita como fases principales, y apatita, turmalina, circón e ilmenita como accesorios.

\section{Metapsamitas y esquistos cuarzo-micáceos con} andalucita y cordierita (Dif-56, Gar-36 y Dif-57, Apéndice, figura 2c). Son de colores grises a ocreamarillentos, con tamaños de grano entre $0,1 \mathrm{y} 1 \mathrm{~mm}$. Las metapsamitas muestran texturas granolepidoblásticas, mientras que los esquistos presentan una textura lepidoblástica con esporádicas intercalaciones de bandas granoblásticas. La composición mineralógica principal es cuarzo, plagioclasa, feldespato potásico, muscovita, cordierita y andalucita, con circón, apatita, monacita, turmalina, ilmenita y magnetita como fases accesorias.

Gneises (Esp-70, Apéndice, figura 2d). Estas rocas presentan un bandeado gnéisico dado por la alternancia de bandas micáceas de 2 a $5 \mathrm{~cm}$ con otras de composición cuarzo-feldespática de hasta $10 \mathrm{~cm}$ de espesor. A su vez, en sectores localizados, se intercalan bancos o lentes de composición psamítica o esporádicamente de composición calcosilicática, de 10 a $15 \mathrm{~cm}$ de espesor. Los gneises tienen una textura granolepidoblástica, con alternancia de bandas cuarzo-feldespáticas de $2 \mathrm{~mm}$ de espesor y bandas lepidoblásticas conformadas por biotita, muscovita y sillimanita (fibrolita). La asociación mineral es
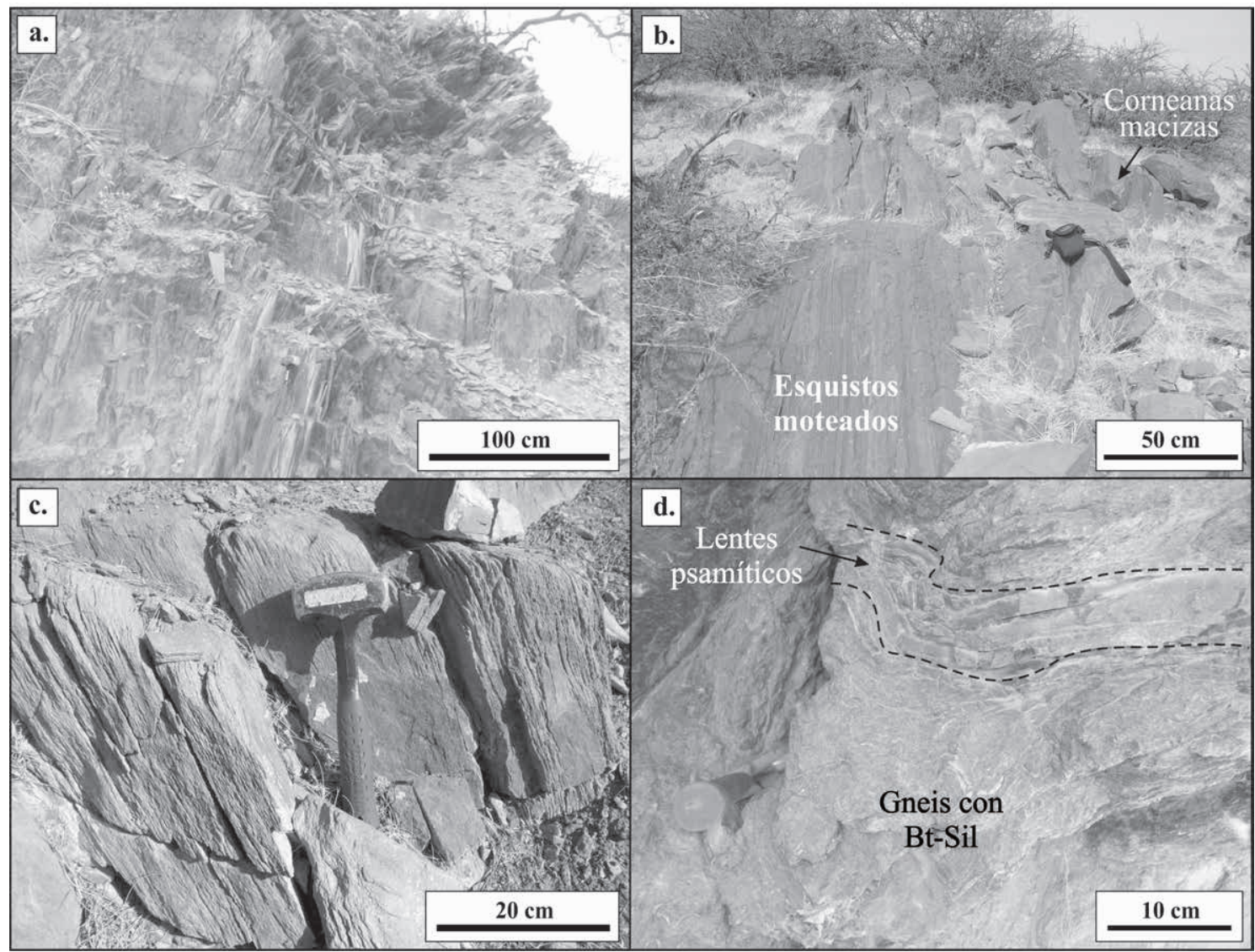

FIG. 2. Fotografías de afloramientos de las distintas litologías analizadas. a. Afloramiento de las filitas en el sector sur de la Sierra de Los Llanos; b. Afloramiento de las rocas corneanas macizas junto a esquistos moteados; c. Detalle de esquistos cuarzo-micáceos con andalucita-cordierita; d. Gneis biotítico-sillimanítico con intercalaciones centimétricas de lentes de composición psamítica. 
cuarzo, plagioclasa, biotita, muscovita y sillimanita como fases principales, y como accesorios, circones incluidos en biotita, apatita y minerales opacos (e.g., ilmenita, magnetita).

\subsection{Composición química}

\subsubsection{Elementos Mayores}

Las muestras de rocas metasedimentarias analizadas registran composiciones geoquímicas equivalentes a psamitas relativamente poco maduras (sublitoarenita y subarcosa) y pelitas, como puede verse en el diagrama de clasificación química de Herron (1988; Fig. 3), el cual muestra las relaciones entre $\mathrm{SiO}_{2} / \mathrm{Al}_{2} \mathrm{O}_{3}$ versus $\mathrm{Fe}_{2} \mathrm{O}_{3} / \mathrm{K}_{2} \mathrm{O}$. Las metapelitas ( $n=5:$ Sab-144, Dif-61, Gar-36, Dif-57 y Esp-70) registran las concentraciones más bajas de $\mathrm{SiO}_{2}$ $(55,4$ a $62,2 \%)$ y más altas de $\mathrm{Al}_{2} \mathrm{O}_{3}(18,4$ a $21,7 \%)$ y $\mathrm{K}_{2} \mathrm{O}(4,5$ a $5,3 \%)$, en contraste con las muestras de metapsamitas ( $n=3$ : Gar-33, Gar-38 y Dif-56) las cuales registran un 85,2 a $87,9 \%$ de $\mathrm{SiO}_{2}, 5,8$ a $6,9 \%$ de $\mathrm{Al}_{2} \mathrm{O}_{3}$ y 1 a $2,4 \%$ de $\mathrm{K}_{2} \mathrm{O}$. Las relaciones de $\mathrm{Al}_{2} \mathrm{O}_{3} /$ $\mathrm{K}_{2} \mathrm{O}$ son altas en todas las muestras, con valores de 2,9 a 6,1 en metapsamitas y 3,8 a 4,1 en metapelitas, lo que indica la presencia significativa de minerales aluminosos en ambos casos. Estas diferencias en composición resultan consistentes con la variación de la asociación mineralógica de los protolitos, como puede observarse en la figura 4 al comparar distintos elementos con el contenido de $\mathrm{SiO}_{2}$. En esta figura se puede observar que las concentraciones de $\mathrm{TiO}_{2}$,

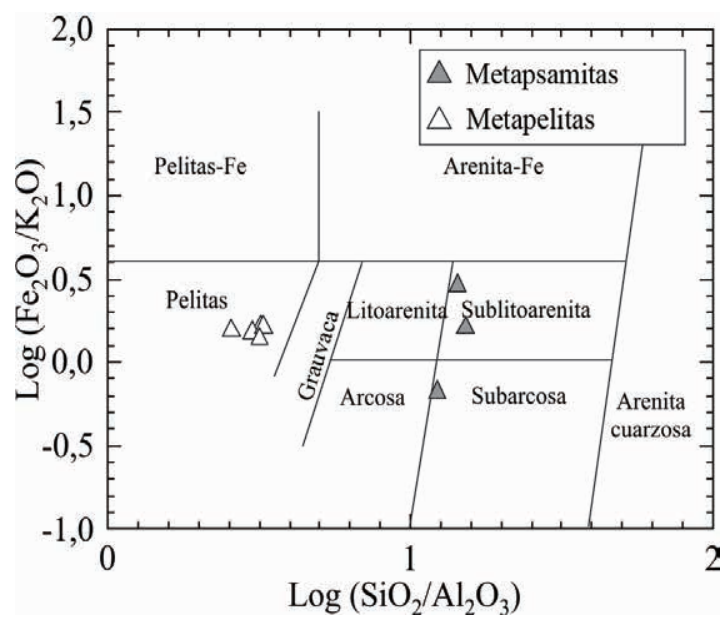

FIG. 3. Clasificación geoquímica de las rocas metasedimentarias del Complejo Metamórfico Los Llanos propuesta por Herron (1988).
$\mathrm{Al}_{2} \mathrm{O}_{3}, \mathrm{Fe}_{2} \mathrm{O}_{3}$ (total), $\mathrm{MgO}$ y $\mathrm{K}_{2} \mathrm{O}$ (indicadores de la abundancia relativa de minerales ferromagnesianos, arcillas y minerales accesorios como ilmenita, hematita, magnetita) muestran una relación lineal y de pendiente negativa con respecto al $\mathrm{SiO}_{2}$, siendo las metapelitas las que presentan mayor concentración de estos elementos. En tanto, el $\mathrm{CaO}$ no muestra variaciones composicionales significativas en relación con la concentración de $\mathrm{SiO}_{2}(0,22-0,64 \% \mathrm{CaO}$ en metapelitas y $0,29-0,85 \% \mathrm{CaO}$ en metapsamitas), mientras que el $\mathrm{Na}_{2} \mathrm{O}$ exhibe una mayor dispersión en metapelitas $\left(0,46-1,90 \% \mathrm{Na}_{2} \mathrm{O}\right)$ en comparación con las metapsamitas (1,15-1,39\%). Por otra parte, las metapelitas contienen mayores concentraciones de $\mathrm{P}_{2} \mathrm{O}_{5}(0,11-0,19 \%)$ que las metapsamitas $(0,08$ $0,12 \%)$, lo que sugiere que parte del contenido de $\mathrm{CaO}$ estaría aportado por apatita, observada petrográficamente, en adición a la plagioclasa.

Al comparar las composiciones de las muestras del CMLL con estándares para pelitas (pelita australiana postarqueana o Post-Archaean Australian shale average, PAAS) y areniscas (Arenisca Cratónica Fanerozoica, ACF), se observa que las mismas resultan parcialmente similares (Figs. 4 y 5). Las metapelitas muestran concentraciones significativamente menores en $\mathrm{CaO}$ con respecto al PAAS, mientras que las metapsamitas se corresponden parcialmente con la composición de la Arenisca Cratónica Fanerozoica (ACF), exhibiendo algunas diferencias importantes señaladas por las menores concentraciones de $\mathrm{CaO}$ y mayores en $\mathrm{Na}_{2} \mathrm{O}$ y $\mathrm{P}_{2} \mathrm{O}_{5}$. A pesar de estas diferencias en álcalis, los valores del Índice de Alteración Química o CIA $\left[\mathrm{Al}_{2} \mathrm{O}_{3} /\left(\mathrm{Al}_{2} \mathrm{O}_{3}+\mathrm{CaO}^{*}+\mathrm{Na}_{2} \mathrm{O}+\mathrm{K}_{2} \mathrm{O}\right)\right.$, donde el $\mathrm{CaO}^{*}$ representa únicamente el perteneciente a los silicatos y corregido según la sugerencia de McLennan, 1993] resultan comparables entre las composiciones estándares (PAAS y ACF) en las muestras del CMLL. Las metapelitas registran valores de CIA de 69 a 76, típico para pelitas como el PAAS (CIA=70), mientras que las metapsamitas tienen valores de 56 a 60 , los que se correlacionan con la composición del ACF (CIA=60).

\subsubsection{Elementos trazas}

En el CMLL, las metapelitas presentan concentraciones de Cs (13-25 ppm), Rb (181-241 ppm), Ba (455-747 ppm) más altas que en las metapsamitas ( $\mathrm{Cs}=3-5 \mathrm{ppm}, \mathrm{Rb}=56-92 \mathrm{ppm}, \mathrm{Ba}=128-302 \mathrm{ppm}$ ), en correspondencia con los contenidos de $\mathrm{K}_{2} \mathrm{O}$. Por otra parte, las metapsamitas exhiben mayores con- 

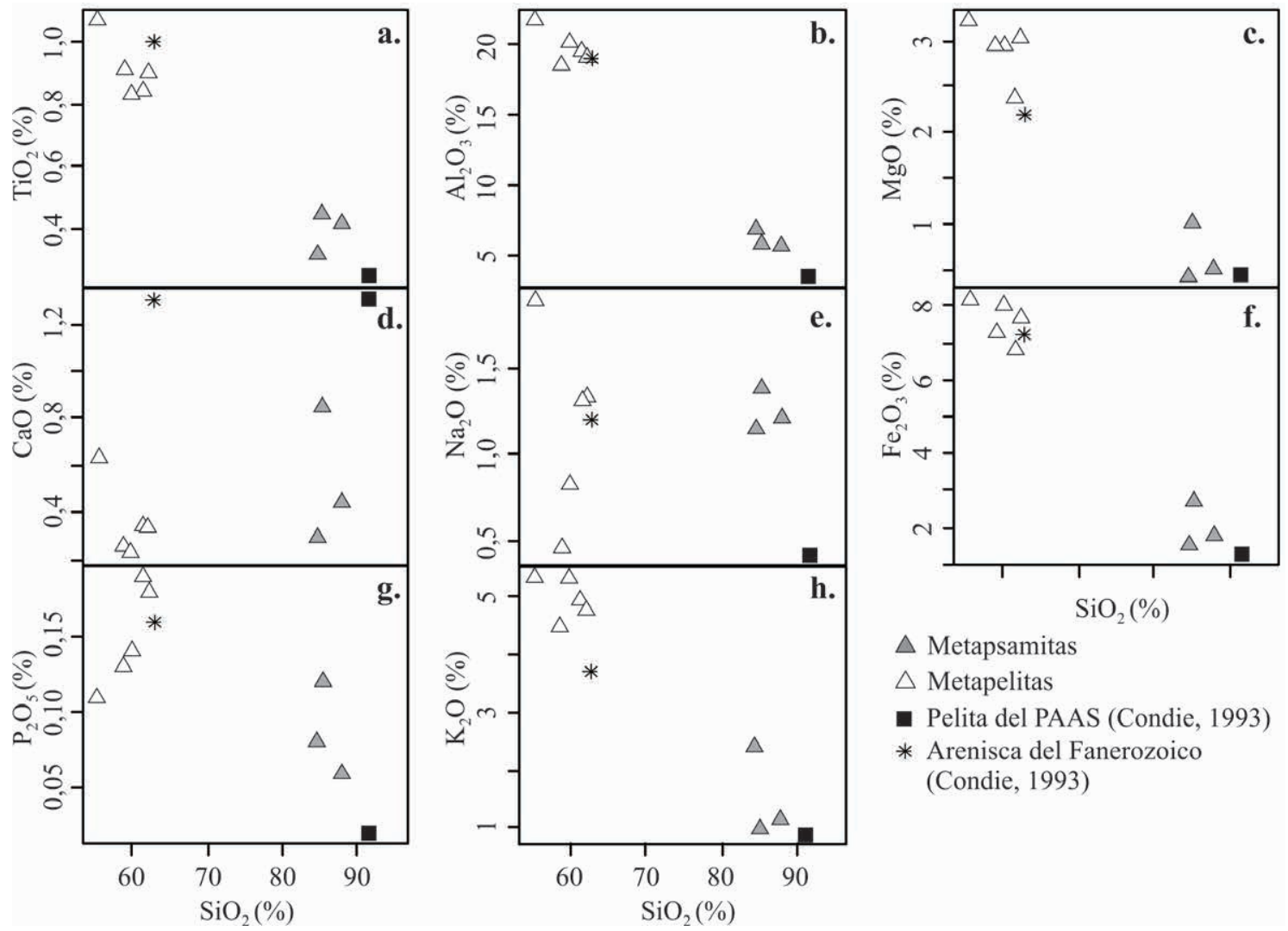

$\triangle$ Metapsamitas

$\triangle$ Metapelitas

- Pelita del PAAS (Condie, 1993)

* Arenisca del Fanerozoico (Condie, 1993)

FIG. 4. Diagramas de variación de óxidos de elementos mayoritarios en base a $\mathrm{SiO}_{2}$ para muestras de metapelitas y metapsamitas del Complejo Metamórfico Los Llanos.

centraciones en $\mathrm{Zr}$ (265-463 ppm) y Hf (8-12 ppm) que las metapelitas ( $\mathrm{Zr}=139-195 \mathrm{ppm}, \mathrm{Hf}=4-6 \mathrm{ppm})$, mostrando una correlación positiva con el $\mathrm{SiO}_{2}$ pero negativa con el $\mathrm{Al}_{2} \mathrm{O}_{3}$. Esto resulta consistente con la concentración preferencial de minerales pesados, como circón, en las unidades más psamíticas. Las concentraciones de $\mathrm{Rb}$ y Ba muestran una correlación positiva con el $\mathrm{K}_{2} \mathrm{O}$, mientras que las concentraciones medias de $\mathrm{Sr}$ no exhiben diferencias significativas entre metapelitas ( $86,4 \mathrm{ppm}$ ) y metapsamitas ( $88 \mathrm{ppm}$ ), en correspondencia con los bajos contenidos de $\mathrm{CaO}$ de ambos grupos de muestras. Las metapelitas exhiben mayores concentraciones de $\mathrm{U}(4,2-4,6 \mathrm{ppm})$, Y (28-42 ppm), Th (10-24 ppm), Sc (16-22 ppm) que las metapsamitas $(\mathrm{U}=2,5-3,7 \mathrm{ppm}, \mathrm{Y}=21-30 \mathrm{ppm}$, $\mathrm{Th}=2-15 \mathrm{ppm}$ y $\mathrm{Sc}=4,5 \mathrm{ppm})$, lo que indicaría un control por parte de minerales ferromagnesianos y minerales del grupo de las arcillas. En adición, el $\mathrm{Ni}, \mathrm{Nb}, \mathrm{Cr}, \mathrm{Pb}, \mathrm{y} \mathrm{V}$ se concentran preferencialmente en metapelitas $(\mathrm{Nb}=18-22 \mathrm{ppm}, \mathrm{Ni}=39-50$ ppm, $\mathrm{Cr}=74-103$ ppm, $\mathrm{Pb}=21-28$ ppm y $\mathrm{V}=106-185$ $\mathrm{ppm})$ respecto a las metapsamitas $(\mathrm{Nb}=9-10 \mathrm{ppm}$, $\mathrm{Ni}=4-8 \mathrm{ppm}, \mathrm{Cr}=31-61 \mathrm{ppm}, \mathrm{Pb}=14-23 \mathrm{ppm}, \mathrm{y}$ $\mathrm{V}=32-36 \mathrm{ppm})$.

Al comparar la concentración de elementos trazas en metapelitas con la composición del PAAS y la composición promedio de la corteza superior (CCS) propuesta por McLennan (2001), se observa que las mismas resultan semejantes, salvo en el Sr, lo que se correlaciona con las bajas concentraciones de $\mathrm{CaO}$ en metapelitas (Fig. 5a). Por otra parte, las metapsamitas muestran mayores diferencias respecto al ACF con concentraciones mayores en $\mathrm{Rb}$, Sr, U, Sc, Y, Zr y Nb (Fig. 5b), que podrían indicar una mayor participación de fases accesorias en las muestras del CMLL. Además, las composiciones de las metapsamitas exhiben una tendencia a aproximarse a la CCS.

La concentración total de elementos del grupo tierras raras (REE) en las muestras de metapsamitas 

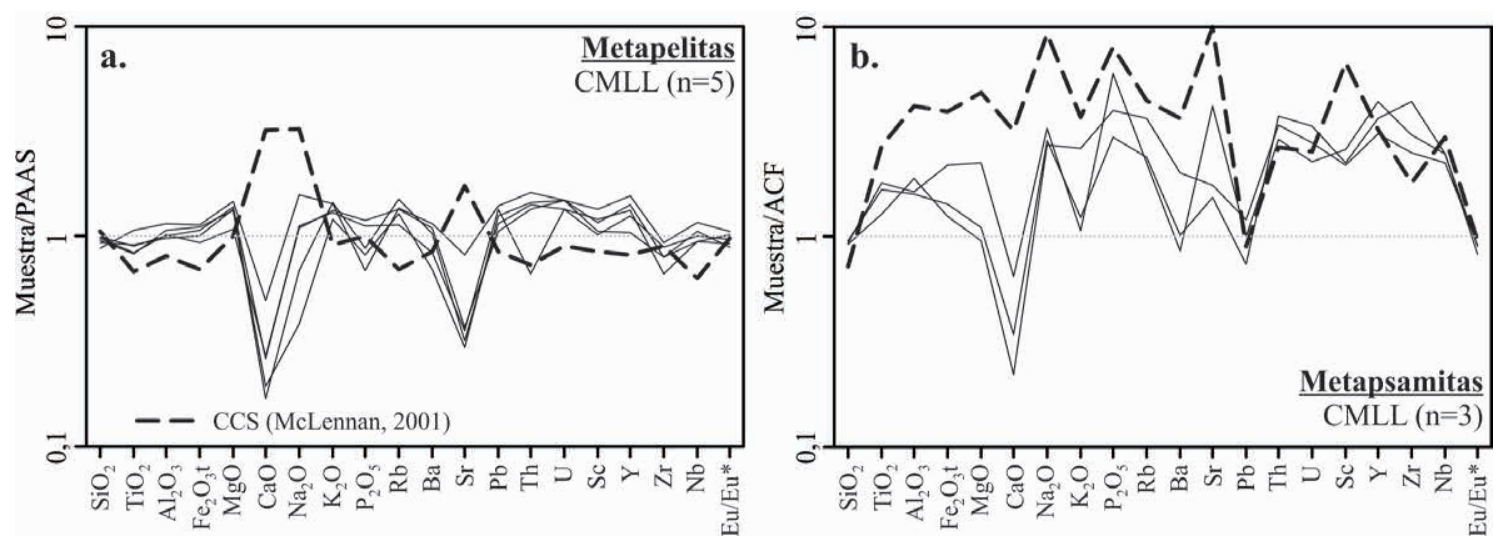

FIG. 5. Diagramas multielementales que proyectan las muestras del Complejo Metamórfico Los Llanos normalizando las metapelitas a la pelita del PAAS (Post-Archaean Australian shale average; Fig. a) y las metapsamitas al ACF (Arenisca Cratónica Fanerozoica; Fig. b). Ver texto para mayor explicación.

varía en el rango de 139 a 185 ppm, mientras que en las metapelitas esta concentración es generalmente mayor con valores de 158 a 309 ppm. En la figura 6 se proyectan los diagramas de REE normalizadas a la composición del condrito ( $\left.\sum \mathrm{REE}=3,476 \mathrm{ppm}\right)$, mostrando además los patrones de composiciones estándares de pelita del PAAS ( $\sum \mathrm{REE}=165 \mathrm{ppm}$; Condie, 1993) y arenisca del $\mathrm{ACF}\left(\sum \mathrm{REE}=45 \mathrm{ppm}\right.$; Condie, 1993), carentes de análisis de Pr, Dy, Ho, Er y Tm, y la composición de la corteza superior (CCS, $\sum$ REE=146 ppm). En general, las metapelitas y metapsamitas muestran patrones de tierras raras similares, con un enriquecimiento preferencial de tierras raras livianas (LREE, serie La-Gd), y un patrón plano para las tierras raras pesadas (HREE, serie $\mathrm{Tb}-\mathrm{Lu}$ ). El patrón de las metapelitas resulta similar al PAAS y al CCS (Fig. 6a), mientras que las metapsamitas presentan un patrón de tierras raras con tendencias similares al ACF, aunque con concentraciones de varios órdenes de magnitud mayor (Fig. 6b). Todas las muestras analizadas presentan anomalías negativas de Eu con un valor promedio de $\mathrm{Eu} / \mathrm{Eu}^{*}$ de 0,60 en metapsamitas y 0,64 en metapelitas (Apéndice), más marcadas que en el PAAS $(0,66)$, ACF $(0,68)$ y en CSS $(0,65)$, en consistencia con los bajos valores de $\mathrm{CaO}$ y $\mathrm{Sr}$ (Fig. 6). El mayor contenido de tierras raras livianas y pesadas en las metapelitas ( $\sum$ LREE=143 a $287 \mathrm{ppm}$, $\sum$ HREE=16 a $22 \mathrm{ppm}$ ) respecto a las metapsamitas ( $\sum$ LREE=128 a $172 \mathrm{ppm}, \sum$ HREE=11 a $15 \mathrm{ppm}$ ) sugiere un enriquecimiento relativo en REE en los sedimentos que puede ser explicado por una mayor concentración de minerales del grupo de las arcillas, óxidos e hidróxidos de hierro y minerales accesorios. Respecto a esto último, uno de los accesorios más probables es la apatita. Sin embargo las concentraciones de REE responden negativamente al incremento en el contenido de $\mathrm{P}_{2} \mathrm{O}_{5}$ en las metapsamitas y en las metapelitas, sugiriendo que este mineral no es la principal fuente de estos elementos. Esto resulta consistente con las interpretaciones de McLennan (1989) y Condie (1991), quienes han indicado que los minerales del grupo de las arcillas son importantes reservorios de LREE, Y, U y Th. En adición, fuentes ígneas de composición félsica pueden aportar LREE, lo cual resulta consistente con la anomalía negativa de Eu.

\subsubsection{Caracterización de áreas fuente}

Considerando el metamorfismo como un proceso esencialmente isoquímico con movilización limitada de elementos, la concentración actual de elementos mayores, trazas y tierras raras reflejaría la composición mineralógica de los sedimentos de los protolitos, la intensidad de los procesos de selección sedimentaria y las posibles áreas fuente de los mismos.

Las relaciones $\mathrm{Rb} / \mathrm{Sr}$ en las metapsamitas $(0,4$ a 1,5$)$ y metapelitas $(1,33$ a 3,49$)$ sugieren que los protolitos sedimentarios registraban grados variables de madurez mineralógica (ver Patocka y Storch, 2004), con una tendencia a la participación de sedimentos más maduros en las metapelitas. Las relaciones Ti/ $\mathrm{Zr}, \mathrm{Mg} \#[\mathrm{Mg} /(\mathrm{Mg}+\mathrm{Fe})]$ y $\mathrm{Cr} / \mathrm{Th}$, las cuales son más altas en metapelitas, y las relaciones $\mathrm{La} / \mathrm{Sc}, \mathrm{Th} / \mathrm{Sc}$ 

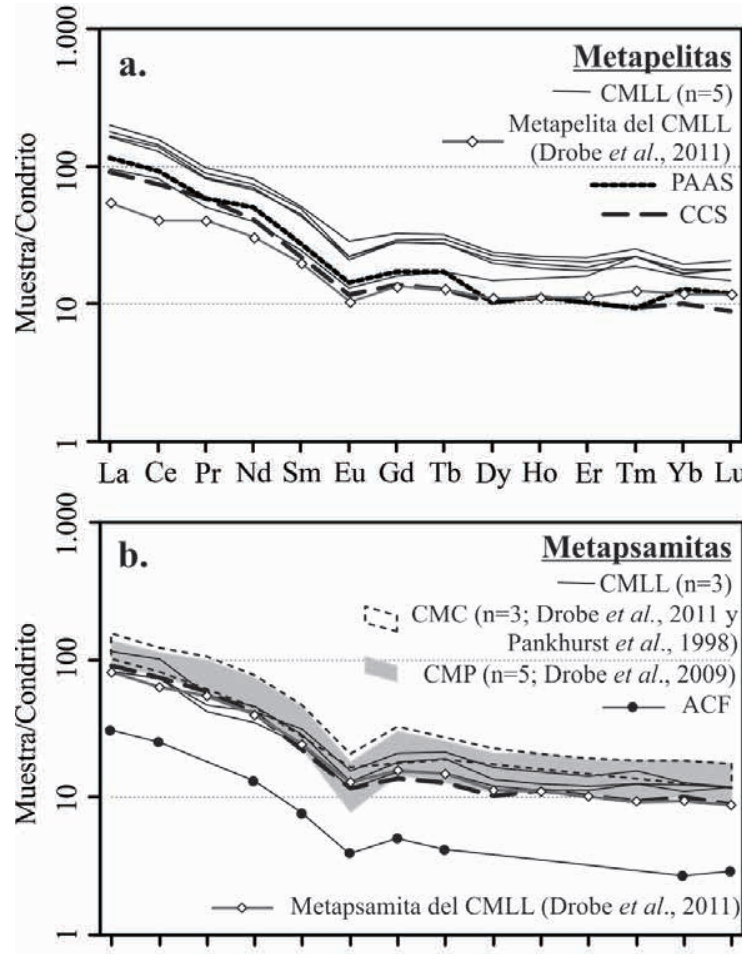

La Ce Pr Nd Sm Eu Gd Tb Dy Ho Er Tm Yb Lu

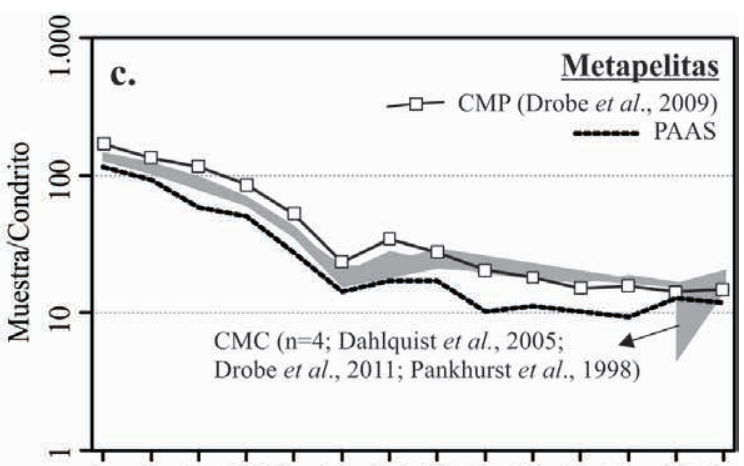

La Ce Pr Nd Sm Eu Gd Tb Dy Ho Er Tm Yb Lu

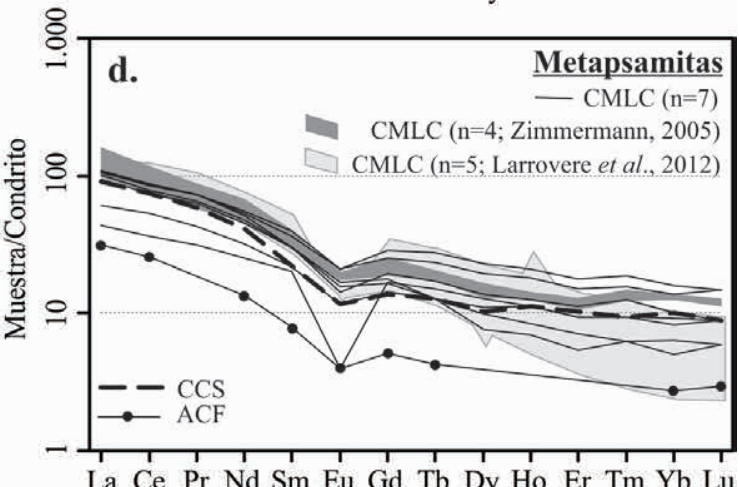

FIG. 6. Distribución de la concentración de elementos del grupo de las tierras raras normalizados a la composición del condrito (análisis de Nakamura, 1974, completado con análisis de Pr, Tb, Ho, Tm de Boynton, 1984) para las muestras de metapelitas (a) y metapsamitas (b) del Complejo Metamórfico Los Llanos (CMLL). Se proyectan, además, las composiciones de muestras del Complejo Metamórfico Chepes (CMC; Pankhurst et al., 1998; Dahlquist et al., 2005; Drobe et al., 2011) y del Complejo Metamórfico Pringles (CMP; Drobe et al., 2009) en (c), y muestras de metapsamitas del Complejo Metamórfico La Cébila (CMLC) de este trabajo (d) junto a otras muestras de metapsamitas publicadas para este complejo (Zimmermann, 2005; Larrovere et al., 2012).

y $\mathrm{V} / \mathrm{Ni}$, que los son en metapsamitas (Tabla 1 y Apéndice) indican una derivación de material más ácido en las psamitas, y de tendencia básica en las pelitas, lo cual resulta consistente con una mayor participación de minerales del grupo de las arcillas en estas últimas. En adición, esto resulta consistente con las mínimas diferencias en los valores medios de $\mathrm{Eu} / \mathrm{Eu}^{*}$ entre muestras de metapsamitas $(0,60)$ y metapelitas $(0,64)$. De esta manera, las muestras clasificadas como metapelitas presentan una composición coherente con la presencia de arcillas y minerales accesorios en el protolito, mientras que las metapsamitas se desarrollaron a partir de sedimentos mayormente enriquecidos en cuarzo, feldespato potásico, y minerales accesorios pesados (e.g., circón), con escasa participación de minerales del grupo de las arcillas. Estas diferencias mineralógicas resultan consistentes con procesos de selección sedimentaria, sin ser necesaria la participación de diferentes áreas fuente.

La impronta geoquímica de las áreas fuente de sedimentos y el contexto geotectónico al cual estarían vinculadas pueden aproximarse a partir de las rocas metapsamíticas, analizando la concentración de elementos HFS (High Field Strength) cuyas relaciones interelementales son herederas de las de la fuente del sedimento dado que no experimentan cambios significativos (elementos inmóviles o de baja movilidad) durante la diagénesis y metamorfismo (e.g., La, Th, Zr, Hf, Sc y Ti; Bathia y Crook, 1986). Elementos como Th y La son útiles para identificar señales de fuentes ígneas félsicas; el Zr y el La reflejan el grado de reciclado cortical o aporte de fuentes silíceas, mientras que otros como Sc y Ti hacen referencia a fuentes máficas (Bathia y Crook, 1986). En la figura 7 se proyectan 
diferentes diagramas de discriminación de ambiente geotectónico propuestos por diferentes autores para rocas de composición psamítica ( $\mathrm{Th} / \mathrm{Sc}$ versus $\mathrm{Zr} / \mathrm{Sc}$ de McLennan et al., 1990; Sc-Th-Zr/10, Th-La-Sc y $\mathrm{La} / \mathrm{Sc}$ vs Ti/Zr de Bathia y Crook, 1986; Hf versus La/Th de Floyds y Leveridge, 1987; los valores de estas relaciones están resumidos en la tabla 1). Los resultados señalan que los protolitos de las unidades metapsamíticas del CMLL se corresponderían con fuentes de tipo continental asociadas a un margen pasivo (las tres muestras se proyectan en el campo D; Figs. 7a, b y d). Las bajas relaciones de Ti/Zr $(5,4-8,4)$, las concentraciones relativamente altas $\mathrm{Hf}(7,9$ a 12,4 ppm) y las altas relaciones de $\mathrm{La} / \mathrm{Sc}$ $(5,2-8,5), \mathrm{Th} / \mathrm{Sc}>1(2,2-3,4)$ y $\mathrm{Zr} / \mathrm{Sc}(60-103)$ indican que los sedimentos originales de las tres muestras de metapsamitas proceden de la erosión de un área fuente enriquecida en componentes félsicos.

\section{Caracterización de los elementos del grupo de las tierras raras y análisis del área fuente de las me- tapsamitas del Complejo Metamórfico La Cébila}

Las muestras de metapsamitas del Complejo Metamórfico La Cébila registran concentraciones de elementos de tierras raras que van desde 85 a $189 \mathrm{ppm}$ con $\sum$ LREE de 74-170 ppm y $\sum$ HREE de 6-19 ppm ( $\mathrm{n}=7$; Apéndice). Los patrones de REE normalizados a condrito (Fig. 6d) muestran un enriquecimiento preferencial en LREE, con una marcada pendiente que tiende a ser horizontal para las HREE (Fig. 6d). Todos los análisis muestran patrones con anomalías negativas de $\mathrm{Eu}$ con valores de $\mathrm{Eu} / \mathrm{Eu}^{*}$ dentro del rango de $0,60-0,72$, con excepción de una muestra que presenta una marcada anomalía con valor de 0,22 (CEB-353). Este rango resulta consistente con las composiciones de la corteza superior $(\mathrm{CCS}=0,65)$ y de la arenisca del Fanerozoico ( $\mathrm{ACF}=0,68)$. El patrón general de REE de las metapsamitas del CMLC resulta similar con la CCS y de varios órdenes de magnitud mayor a la composición de la ACF.

La concentración de elementos HFS en las muestras de metapsamitas del complejo La Cébila (presente trabajo) exhiben bajas relaciones Ti/Zr (2 a 7) y relativamente altas en $\mathrm{Th} / \mathrm{Sc}(0,8-3,6), \mathrm{Zr} / \mathrm{Sc}$ $(20,9-136,8)$ y La/Sc (2-16) como puede verse en las figuras $7 \mathrm{~d}$ y e. Estos valores son compatibles con fuentes de composición félsica o con grados diferentes de reciclado, como fue sugerido por Verdecchia y Baldo (2010) a partir de las relaciones La/Th y los contenidos de $\mathrm{Hf}$ (Fig. 7c). Los diagramas ternarios $\mathrm{Sc}-\mathrm{Th}-\mathrm{Zr} / 10$ y Th-La-Sc exhiben similares comportamientos (Figs. 7a y b), proyectando las composiciones dentro de los campos correspondientes a arco de isla continental (campo B), margen continental activo (campo C) y margen pasivo (campo D). A pesar de que estos diagramas no permiten realizar una clara caracterización del ambiente geotectónico de las fuentes, los mismos señalan una baja participación de fuentes de composición básica.

\section{Discusión}

\subsection{Correlación con el Complejo Metamórfico Chepes}

Las rocas plutónicas y metamórficas de la Sierra de Los Llanos han sido consideradas como la continuación hacia el norte de los complejos ígneo y metamórfico de la Sierra de Chepes (CMC), debido a las similitudes petrológicas, geoquímicas y estructurales (Pankhurst et al., 1998; Pascua et al., 1998). Las unidades metapsamíticas y metapelíticas de la Sierra de Chepes (Pankhurst et al., 1998; Dahlquist et al., 2005; Drobe et al., 2011) exhiben patrones de REE similares al CMLL, mostrando marcadas anomalías de Eu (Figs. 6b, c y d). Drobe et al. (2011) sugieren que a partir de las diferencias composicionales entre metapsamitas del CMC $(\mathrm{n}=2 ; \mathrm{Ti} / \mathrm{Zr}=14-22, \mathrm{La} / \mathrm{Sc}=3,7-5,2, \mathrm{Hf}=5,3-8,3 \mathrm{ppm}$, $\mathrm{Th} / \mathrm{Sc}=1,1-1,7 \mathrm{y} \mathrm{Zr} / \mathrm{Sc}=16,1-28)$ y la metapsamita del CMLL $(\mathrm{Ti} / \mathrm{Zr}=31, \mathrm{La} / \mathrm{Sc}=2,8, \mathrm{Hf}=3,1 \mathrm{ppm}$, $\mathrm{Th} / \mathrm{Sc}=0,8$ y $\mathrm{Zr} / \mathrm{Sc}=11,8)$, los protolitos del $\mathrm{CMC}$ representarían sedimentos de grados variables de madurez procedentes de fuentes con mayor participación de un arco de isla continental mientras que en Los Llanos habría participación de fuentes máficas. Sin embargo, los análisis del CMLL mostrados en el presente trabajo resultan diferentes en relación con el publicado por estos autores, como puede observase en la figura 7 , sugiriéndose la participación de fuentes de composición félsica o con mayor grado de reciclado cortical para la Sierra de Los Llanos. Aunque existen estas diferencias composicionales entre las muestras del CMLL y $\mathrm{CMC}$, las relaciones de $\mathrm{Zr} / \mathrm{Sc}$ versus $\mathrm{Th} / \mathrm{Sc}$, Hf versus $\mathrm{La} / \mathrm{Th}$ y La/Sc versus Ti/Zr (Fig. 7c, d y e) resultan compatibles con fuentes corticales asociadas a un ambiente geotectónico de arco continental, según los diagramas de discriminación geotectónica. 

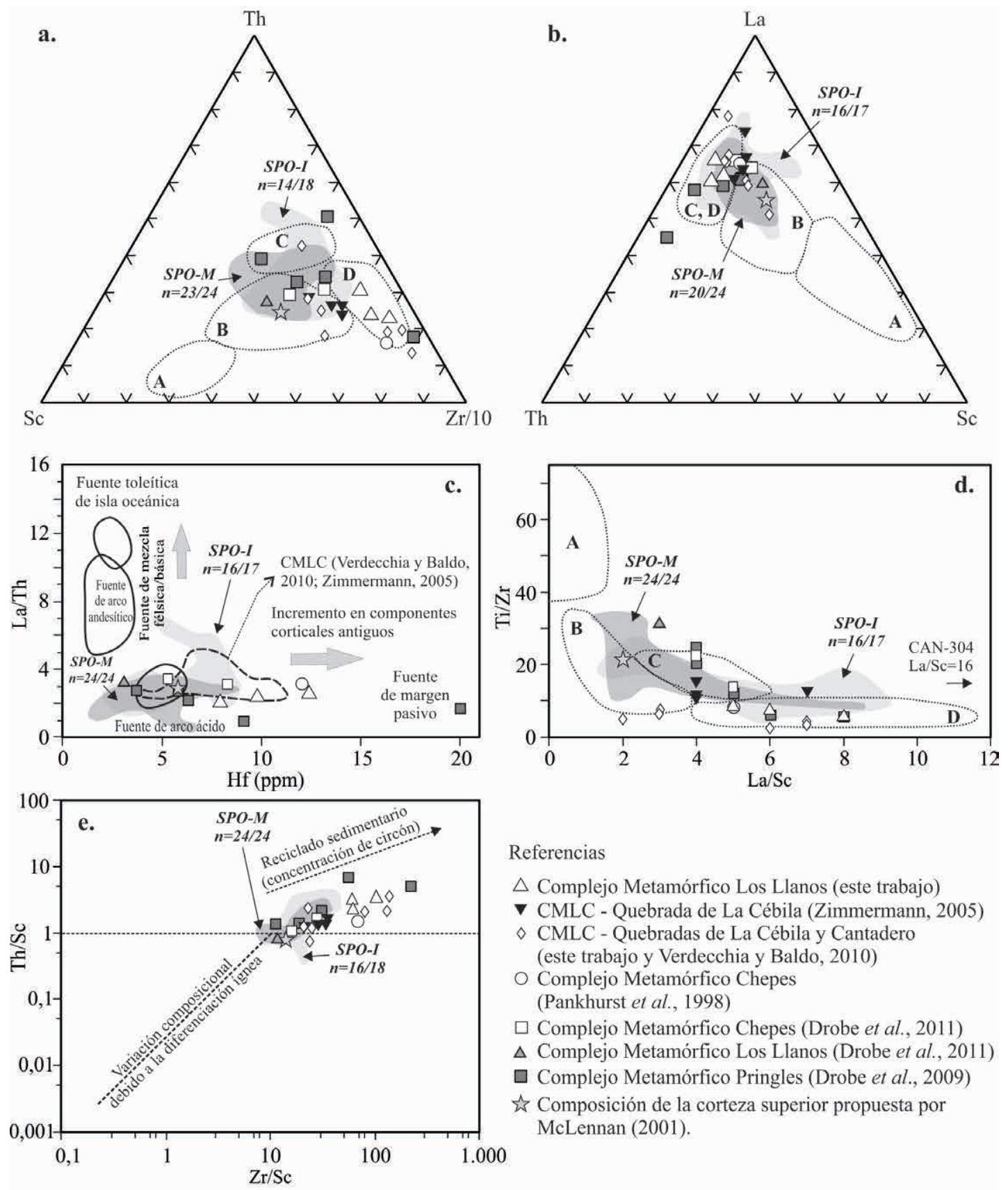

Referencias

$\triangle$ Complejo Metamórfico Los Llanos (este trabajo)

CMLC - Quebrada de La Cébila (Zimmermann, 2005)

$\diamond$ CMLC - Quebradas de La Cébila y Cantadero (este trabajo y Verdecchia y Baldo, 2010)

O Complejo Metamórfico Chepes (Pankhurst et al., 1998)

$\square$ Complejo Metamórfico Chepes (Drobe et al., 2011)

$\Delta$ Complejo Metamórfico Los Llanos (Drobe et al., 2011)

$\square$ Complejo Metamórfico Pringles (Drobe et al., 2009)

放 Composición de la corteza superior propuesta por McLennan (2001).

FIG. 7. Diagramas de ambientes geotectónicos que proyectan las muestras de metapsamitas de los complejos metamórficos Los Llanos y Chepes junto con las muestras del Complejo Metamórfico La Cébila (este trabajo y datos de Verdecchia y Baldo, 2010). (a-b) diagramas ternarios Sc-Th-Zr/10 y Th-La-Sc de Bhatia y Crook (1986), (c) diagrama Hf versus La/Th de Floyds y Leveridge, (1987), (d) diagrama La/Sc versus Ti/Zr de Bhatia y Crook (1986), (e) diagrama Th/Sc versus Zr/Sc de McLennan et al. (1990). También se proyectan las muestras de metapsamitas del CMLC publicadas por Zimmermann (2005), del CMC (Pankhurst et al., 1998; Dahlquist et al., 2005; Drobe et al., 2011), y del CMP (Drobe et al., 2009). Las áreas señaladas como SPO-M y SPO-I están definidas por la distribución de dos grupos de muestras de rocas metasedimentarias ( $n=24)$ e ígneas metaluminosas $(n=18)$ del Cámbrico temprano respectivamente, representativas del arco Pampeano en las Sierras Pampeanas Orientales (SPO) (datos extraídos de Rapela et al., 1998; Gosen et al., 2009; Iannizzotto et al., 2013). Los campos en las figuras a, b y d son: arco de isla oceánico (A), arco de isla continental (B), margen continental activo (C) y margen pasivo (D). 
Edades U-Pb SHRIMP en circones detríticos del CMC y del CMLL aportados por Drobe et al. (2011), Sims et al. (1998) y Casquet et al. (2012) permiten acotar la edad máxima de sedimentación al Cámbrico medio (pico de edad más joven $c a$. $520 \mathrm{Ma}$ ), indicando una sedimentación posterior a la orogénesis Pampeana. La edad mínima de sedimentación se sitúa en $c a$. $480 \mathrm{Ma}$, que corresponde a la edad de cristalización del granito anatéctico Tuaní (TUA-1029, ca. 479 \pm 10 Ma; Pankhurst et al., 2000, ver ubicación en figura 1a), derivado de la anatexis de las rocas metasedimentarias de Chepes (Dahlquist et al., 2005). Esto es reforzado por las edades de cristalización U-Pb SHRIMP en circones de rocas que intruyen al CMC y al CMLL como la Granodiorita Chepes de edad 486-483 Ma, y la unidad Asperezas localizada en el extremo sur de la Sierra de Los Llanos y de edad de $477 \pm 4 \mathrm{Ma}$ (Pankhurst et al., 1998, 2000). De esta manera, las edades de herencia y cristalización permiten acotar la sedimentación de los protolitos de las rocas metasedimentarias de Chepes y Los Llanos entre el Cámbrico medio y el Ordovícico Inferior ( $c a$. 520-480 Ma). En ambas sierras, las rocas metasedimentarias contienen, además de circones detríticos paleozoicos y neoproterozoicos, circones detríticos paleoproterozoicos con edades entre 2.020-2.260 Ma, típicas del Cratón del Río de La Plata (Rapela et al., 2007), y que no se reconocen en los sedimentos más antiguos o contemporáneos a la orogenia Pampeana (Rapela et al., 2007; Verdecchia et al., 2011). Esto sugiere que el Cratón del Río de La Plata y otros terrenos situados más al este se constituyeron como áreas fuente solo después de la orogénesis Pampeana (Verdecchia et al., 2011; Casquet et al., 2012).

\subsection{Correlación con otros complejos metamór- ficos de las Sierras Pampeanas e implicancias geotectónicas}

Los protolitos de las rocas metasedimentarias de las sierras de Chepes y de Los Llanos podrían ser equivalentes a otros complejos metasedimentarios de las Sierras Pampeanas Orientales con protolitos pospampeanos afectados por la orogénesis Famatiniana. Se destacan las rocas metasedimentarias de los complejos metamórficos La Cébila (CMLC, provincia de La Rioja y Catamarca) y de Pringles (CMP, provincia de San Luis), ubicadas al noroeste y sureste de las sierras de Los Llanos-Chepes respec- tivamente (Fig. 1). La edad de sedimentación de los protolitos del CMLC ha sido asignada al Ordovícico Inferior sobre la base de braquiópodos preservados en cuarcitas en zona de alto grado metamórfico (Verdecchia et al., 2007). Las rocas de este complejo muestran patrones de edades de circones detríticos similares a los de los CMLL y CMC, registrando el pico de edades más jóvenes en 520-530 Ma, correspondiente a la orogénesis Pampeana, y edades típicas del Cratón del Río de La Plata ( $c a .2,1 \mathrm{Ga}$; Finney et al., 2003, 2004; Rapela et al., 2007; Adams et al., 2011; Verdecchia et al., 2011). Por otra parte, las edades de sedimentación máxima y mínima de los protolitos del Complejo Metamórfico Pringles (CMP) están acotadas por la edad de herencia en circones detríticos $c a$. $530 \mathrm{Ma}$ (edad U-Pb SHRIMP publicada por Sims et al., 1998) y la edad del pico metamórfico de ca. $498 \mathrm{Ma}$ (calculada en bordes de circones por Steenken et al., 2006) respectivamente, limitando así la edad de sedimentación al intervalo Cámbrico medio a tardío.

En la figura $6 \mathrm{~d}$ se proyectan las concentraciones de REE de las metapsamitas del CMLC presentadas en este trabajo y las publicadas por Zimmermann (2005; $\sum$ LREE=146-224 ppm; $\sum$ HREE $=12-14$ $\mathrm{ppm} ; \mathrm{Eu} / \mathrm{Eu}^{*}=0,57-0,74$ para $\mathrm{n}=4$ ) y Larrovere et al. (2012; $\sum$ LREE $=145-233 ; \sum$ HREE $=5-17$ ppm; Eu/ $\left.\mathrm{Eu}^{*}=0,29-0,64\right)$. En general, estas rocas metapsamíticas presentan patrones similares a los registrados en los complejos metamórficos Los Llanos, Chepes y Pringles, pero con un rango composicional mayor (Fig. 6a-c). Estas diferencias pueden estar señalando variaciones del control sedimentario en la concentración preferencial de minerales enriquecidos en estos elementos como circón y apatita dentro de las unidades del CMLC.

La concentración de elementos HFS en las muestras de metapsamitas del CMLC no exhiben una clara discriminación del ambiente geotectónico de las fuentes, pero señalan una significativa influencia de fuentes de composición félsicas o más evolucionadas con baja participación de fuentes de composición básica (Fig. 7), como fuese interpretado por varios autores (Zimmermann, 2005; Verdecchia y Baldo, 2010; Larrovere et al., 2012) de manera similar en el CMLL. Por otra parte, los análisis del CMP publicados por Drobe et al. (2009) resultan comparables al CMC y ambos exhiben pendiente negativa en la relación $\mathrm{Ti} / \mathrm{Zr}$ versus $\mathrm{La} / \mathrm{Sc}$ a diferencia del CMLC y el CMLL. En este sentido, López de Luchi et al. 
(2003) y Drobe et al. (2009) han sugerido fuentes de origen mixto para las rocas metasedimentarias del CMP compatibles con ambiente de margen continental activo o arco de isla continental. A pesar de las diferencias composicionales mostradas en los análisis de metapsamitas de los diferentes complejos estudiados, es importante destacar que las señales geoquímicas no permiten hacer una correspondencia con un determinado tipo geotectónico y que las tendencias en las variaciones en las relaciones entre $\mathrm{Ti}, \mathrm{Zr}, \mathrm{Hf}, \mathrm{La}, \mathrm{Th}$ y Sc hacia fuentes más félsicas o evolucionadas indican que la composición de los protolitos estaría mayormente controlada por los procesos sedimentarios y no por la heterogeneidad composicional de las fuentes.

Aunque los protolitos sedimentarios de Pringles y de Los Llanos-Chepes (Cámbrico medio a tardío) resulten ser, en principio, algo más antiguos que $\mathrm{La}$ Cébila (Ordovícico Inferior), todos estos muestran rasgos geoquímicos parecidos, además de patrones de edades de herencia en circones idénticos (Sims et al., 1998; Steenken et al., 2006; Drobe et al., 2009; Verdecchia et al., 2011; Casquet et al., 2012). Esto sugiere que las cuencas sedimentarias del Cámbrico medio al Ordovícico Inferior habrían compartido áreas de aporte similares de tipo margen pasivo (basamento continental extenso) y arco magmático félsico, con diferente participación de componentes máficos en consistencia con Drobe et al. (2011), y apoyándose, además, con la evidencia que arrojan los circones detríticos. La presencia de circones 'pampeanos' indica que las rocas ígneas volcánicas a plutónicas, mayoritariamente intermedias a ácidas, vinculadas a esta orogénesis (Sierras de Córdoba, Sierra NorteAmbargasta; Rapela et al., 1998; Siegesmund et al., 2010; Iannizzotto et al., 2013; Fig. 1) fueron una importante fuente de circones detríticos y demás material clástico que conformaron los protolitos de los complejos metamórficos analizados (Fig. 8); esto es consistente con las interpretaciones de Steenken et al. (2011). La existencia de una fuente ubicada en el ámbito del cinturón orogénico Pampeano implica que las rocas metasedimentarias pertenecientes al mismo (Formación Puncoviscana y equivalentes, de edad Neoproterozoico-Cámbrico Inferior; Escayola et al., 2011) debieron de ser canibalizadas y recicladas. Ello explicaría los circones detríticos con edades neoproterozoicas brasilianas (570 a $680 \mathrm{Ma}$ ) que se encuentran también en las rocas metasedimentarias pospampeanas (Collo et al., 2009; Verdecchia et al.,

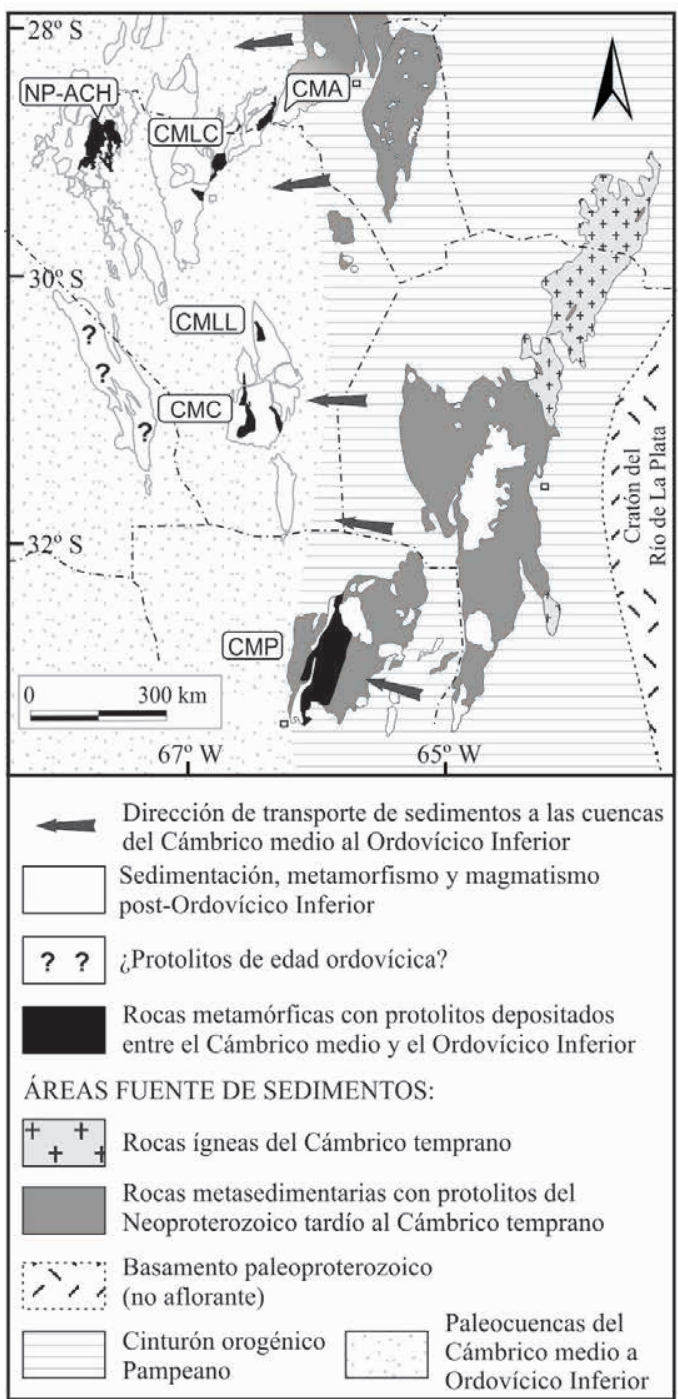

FIG. 8. Mapa geológico sintético de las Sierras Pampeanas Orientales de Argentina ubicando los afloramientos de rocas metasedimentarias, cuyos protolitos estarían vinculados a cuencas desarrolladas entre el Cámbrico medio y Ordovícico Inferior (ver texto para mayores detalles). El basamento pre- a sinorogenia Pampeana se localiza principalmente en el sector este de la región. El mismo habría sido la principal área de aporte de sedimentos de estas cuencas. Abreviaciones: Formaciones Negro Peinado y Achavil (NP-ACH); Complejo Metamórfico Ambato (CMA).

2011; Casquet et al., 2012) y que son característicos de la Formación Puncoviscana. La existencia en las rocas metasedimentarias pospampeanas de la Formación Achavil (Collo et al., 2009) y los complejos metamórficos Los Llanos y La Cébila, 
de poblaciones de circones con edades paleoproterozoicas típicas del Cratón del Río de La Plata ( $c a$. $2,1 \mathrm{Ga}$ ) y otras de $c a .1,8 \mathrm{Ga}$ que no son típicas del mismo, indica además la participación de áreas fuente más distales, ubicadas al este del cinturón Pampeano. Estas fuentes se ubicarían en el propio Cratón del Río de la Plata y en terrenos ubicados más al este del mismo, hoy día ubicados en el margen suroccidental de África, como podría ser la faja orogénica Gariep (Casquet et al., 2012). De esta manera, las cuencas pospampeanas habrían tenido una extensa y compleja área de aporte.

Desde un punto de vista químico, las rocas ígneas y metasedimentarias del cinturón Pampeano (SPO-I y SPO-M respectivamente, en figura 7) muestran relaciones entre $\mathrm{Ti}, \mathrm{Zr}, \mathrm{Hf}, \mathrm{La}$, Th y Sc (Fig. 7) similares a los sedimentos de los complejos metamórficos Los Llanos, Chepes, Pringles y La Cébila, aunque con mayor contenido de componentes máficos, como es sugerido por las relaciones $\mathrm{Ti} / \mathrm{Zr}$ y La/Sc (Fig. 7d) y $\mathrm{Th} / \mathrm{Sc}$ y $\mathrm{Zr} /$ Sc (Fig. 7e), y los diagramas de discriminación geotectónica de Bathia y Crook (1986; Figs. 7a y b). Estas diferencias son imputables a los procesos sedimentarios de reciclado que afectaron a las rocas del basamento pampeano, que han dado como resultado protolitos con una mayor madurez mineralógica para las cuencas pospampeanas.

Rocas metasedimentarias depositadas también en el Cámbrico medio al Ordovícico Inferior se han reconocido en otros lugares de las Sierras Pampeanas Orientales. Tal es el caso del Complejo Metamórfico Ambato (CMA, Verdecchia et al., 2007, 2011; Fig. 1a) y las formaciones Negro Peinado y Achavil (NP-ACH, Collo et al., 2009; Fig. 1a), caracterizadas por registrar picos de edades de herencia correspondientes a la orogenia Pampeana (ca. 530-520 Ma) y ausencia de edades detríticas del Ordovícico. Edades paleoproterozoicas del rango 2,02-2,26 Ga son únicamente registradas en la Formación Achavil que sería probablemente equivalente por ello a las rocas metasedimentarias estudiadas en este trabajo. Verdecchia et al. (2011) han señalado que el Cratón del Río de La Plata no era un área de aporte durante el período de sedimentación de los protolitos del CMA y de la Formación Negro Peinado. La edad de sedimentación de la Formación Achavil ha sido acotada al Cámbrico tardío (Collo et al., 2009), mientras que las edades de sedimentación de los protolitos del CMA y de la Formación Negro Peinado han sido limitadas al Cámbrico medio (Collo et al., 2009; Verdecchia et al., 2011). Estas tres unidades fueron vinculadas a cuencas de antepaís de larga duración dentro de un contexto geotectónico posorogenia Pampeana (Collo et al., 2009; Verdecchia et al., 2011). Más recientemente, Casquet et al. (2012) sugieren que las unidades metasedimentarias que contienen herencias del Cratón del Río de La Plata (2,02-2,26 Ga) como el CMLC y la Formación Achavil, por ende más modernas que el CMA y la Formación Negro Peinado, corresponden a una sedimentación asociada a una amplia plataforma siliciclástica desarrollada en el margen suroccidental de Gondwana, que habría incluido también unidades sedimentarias que afloran en el noroeste de Argentina (Grupo Mesón). En adición, la impronta geoquímica y el registro geocronológico de las unidades metasedimentarias de los CMLC, CMLL, CMC y CMP resultan consistentes con este último modelo, extendiendo regionalmente el funcionamiento de estas cuencas hasta el Ordovícico Inferior (Tremadociano).

\section{Conclusiones}

La señal geoquímica de las rocas metasedimentarias del Complejo Metamórfico Los Llanos permite caracterizar a los tipos litológicos de estas unidades como los equivalentes metamórficos de pelitas y areniscas de madurez intermedia (sublitoarenitas y subarcosas). Las áreas de aporte de los componentes clásticos se corresponden con fuentes recicladas $\mathrm{y} / \mathrm{o}$ enriquecidas en componentes félsicos, y registran además procesos de selección durante el transporte con acumulación de minerales pesados (especialmente circón). Esta signatura geoquímica ha sido también detectada por otros autores en las unidades derivadas del Complejo Metamórfico Pringles (Cámbrico medio a tardío) y el Complejo Metamórfico La Cébila (Ordovícico Inferior) y permite suponer procesos y áreas fuente similares para todos ellos. La información aportada es consistente con la propuesta de postular como probable área fuente de estos sedimentos a los complejos ígneos y metamórficos de la Orogenia Pampeana (Cámbrico inferior), además del Cratón del Río de La Plata, y la existencia de una extensa plataforma continental que bordeaba el margen suroccidental de Gondwana durante el Cámbrico medio al Ordovícico temprano. 


\section{Agradecimientos}

Agradecemos al Fondo para la Investigación Científica y Tecnológica, al Consejo Nacional de Investigaciones Científicas y Técnicas y a la Universidad Nacional de Córdoba por brindar el financiamiento para realizar este trabajo, mediante los proyectos FONCyT Bicentenario PICT-1200, CONICET PIP No 112-201101-00229 y SECyT-UNC 2010/2011. Agradecemos a la Dra. López de Luchi y al Dr. Steenken, por las observaciones realizadas durante el proceso de arbitraje, los cuales han mejorado sustancialmente este trabajo.

\section{Referencias}

Adams, C.; Miller, H.; Aceñolaza, F.; Toselli,A.; Griffin, W. 2011. The pacific gondwana margin in the late neoproterozoic-early Paleozoic: detrital zircon U-Pb ages from metasediments in Northwest Argentina reveal their maximum age, provenance and tectonic setting. Gondwana Research 19 (1): 71-83.

Armstrong-Altrina, J.; Verma, S. 2005. Critical evaluation of six tectonic setting discrimination diagrams using geochemical data of Neogene sediments from known tectonic settings. Sedimentary Geology 177: 115-129.

Bhatia, M.; Crook, K. 1986. Trace element characteristics of graywackes and tectonic setting discrimination of sedimentary basins. Contributions to Mineralogy and Petrology 92: 181-193.

Boynton, W.V. 1984. Geochemistry of the rare earth elements: meteorites studies. In Rare Earth Element Geochemistry (Henderson, P.; editor). Elsevier: 63-114.

Casquet,C.;Rapela,C.;Pankhurst,R.;Baldo,E.; Galindo,C.; Verdeccia, S.; Dahlquist, J.; Murra, J.; Fanning, M. 2012. A post Pampean Middle to Late Cambrian siliciclastic platform on the proto-Andean margin of Gondwana and its paleogeographical implications. Simposio de Geología de la Cordillera de Los Andes y su antepaís. In Congreso Geológico de España, No. 8. Geo-Temas 13: 1852-1855. España.

Collo, G.; Astini, R.; Cawood, P.; Buchan, C.; Pimentel, M. 2009. U-pb detrital zircon ages and Sm-Nd isotopic features in low-grade metasedimentary rocks of the Famatina belt: implications for late neoproterozoicearly palaeozoic evolution of the proto-Andean margin of Gondwana. Journal of the Geological Society of London 116: 1-17.

Condie, K. 1991. Another look at rare earth elements in shales. Geochimica et Cosmochimica, Acta 55: 2527-2531.
Condie, K. 1993. Chemical composition and evolution of the upper continental crust: Contrasting results from surface samples and shales. Chemical Geology 104: 1-37.

Dahlquist, J.; Rapela, C.; Baldo, E. 2005. Cordieritebearing s-type granitoids in the Sierra de Chepes (Sierras Pampeanas): petrogenetic implications. Journal of South American Earth Sciences 20:231-251.

Dahlquist, J.; Alasino, P.; Eby, N.; Galindo, C.; Casquet, C. 2010. Fault controlled Carboniferous A-type magmatism in the proto-Andean foreland (Sierras Pampeanas, Argentina): geochemical constraints and petrogenesis. Lithos 115: 65-81.

Drobe, M.; López de Luchi, M.; Steenken, A.; Frei, R.; Naumann, R.; Siegesmund, S.; Wemmer, K. 2009. Provenance of the late Proterozoic to early Cambrian metaclastic sediments of the Sierra de San Luis (Eastern Sierras Pampeanas) and cordillera Oriental, Argentina. Journal of South American Earth Sciences 28 (3): 239-262.

Drobe, M.; López de Luchi, M.; Steenken,A.; Wemmer, K.; Naumann, R.; Frei, R.; Siegesmund, S. 2011. Geodynamic evolution of the Eastern Sierras Pampeanas (central Argentina) based on geochemical, Sm-Nd, $\mathrm{Pb}-\mathrm{Pb}$ and shrimp data. In International Journal Earth Sciences 100: 631-657. doi: 10.1007/s00531010-0593-3.

Escayola, M.; Pimentel, M.; Armstrong, R. 2007. Neoproterozoic backarc basin: sensitive high-resolution ion microprobe $\mathrm{U}-\mathrm{Pb}$ and $\mathrm{Sm}-\mathrm{Nd}$ isotopic evidence from the Eastern Pampean Ranges, Argentina. Geology 35: 495-498.

Escayola, M.P.; van Staal, C.R.; Davis, W.J. 2011. The age and tectonic setting of the Puncoviscana Formation in northwestern Argentina: An accretionary complex related to Early Cambrian closure of the Puncoviscana Ocean and accretion of the Arequipa-Antofalla block. Journal of South American Earth Sciences 32: 495-498.

Finney, S.; Gleason, J.; Gehrels, G.; Peralta, S.;Aceñolaza, G. 2003. Early Gondwanan connection for the Argentine Precordillera terrane. Earth and Planetary Science Letters 205 (3-4): 349-359.

Finney, S.; Gleason, J.; Gehrels, G.; Peralta, S.;Aceñolaza, G. 2004. Corrigendum to 'Early Gondwana connection for the Argentine Precordillera terrane'. Earth and Planetary Science Letters 219: p. 413.

Floyd, P.; Leveridge, B. 1987. Tectonic environment of the Devonian Gramscatho basin, south Cornwall: framework mode and geochemical evidence from 
turbiditic sandstones. Journal of the Geological Society of London 144: 531-542.

Gosen, W. von; Buggisch, W.; Prozzi, C. 2009. Provenance and geotectonic setting of late Proterozoic-early Cambrian metasediments in the Sierras de Córdoba and Guasayán (western Argentina): A Geochemical Approach. Neues Jahrbuch für Geologie und Paläontologie Abhandungen 251: 257-284.

Herron, M.M. 1988. Geochemical classification of terrigenous sands and shales from core or log data. Journal of Sedimentary Petrology 58 (5): 820-829.

Iannizzotto, N.; Rapela, C.; Baldo, E.; Galindo, C.; Fanning, C.; Pankhurst, R. 2013. The Sierra NorteAmbargasta batholith: late Ediacaran early Cambrian magmatism associated with Pampean transpressional tectonics. Journal of South American Earth Sciences 42: 127-143.

Larrovere, M.; De Los Hoyos, C.; Grosse, P. 2012. Los complejos metamórficos del retro-arco Famatiniano (noroeste deArgentina): caracterización geoquímica e isotópica de sus protolitos e implicancias geotectónicas. Revista Mexicana de Ciencias Geológicas 29 (3): 676-695.

López de Luchi, M.G.; Cerredo, M.E.; Siegesmund, S.; Steenken, A.; Wemmer, K. 2003. Provenance and tectonic setting of the protoliths of the metamorphic complexes of Sierra de San Luis. Revista de la Asociación Geológica Argentina 58 (4): 525-540.

Limarino, C.; Poma, S. 1999. Descripción geológica de la hoja 3166-i, Chamical, provincia de La Rioja. Servicio Geológico Nacional: 35 p. Buenos Aires.

McLennan, S. 1989. Rare Earth elements in sedimentary rocks: influence of provenance and sedimentary process. In Geochemistry and mineralogy of rare Earth elements, Mineralogical Society of America (Lipin, B.; Mckay, G.; editors). Reviews in Mineralogy 21: 169-200.

McLennan, S. 1993. Weathering and Global Denudation. The Journal of Geology 101: 295-303.

McLennan, S. 2001. Relationship between the trace element composition of sedimentary rocks and upper continental crust. Geochemistry, Geophysics, Geosystems 2: 1525-2027.

McLennan, S.; Taylor, S.; McCulloch, M.; Maynard, J. 1990. Geochemical and Nd-Sr isotopic composition of deep-sea turbidites: crustal evolution and plate tectonic associations. Geochimica et Cosmochimica Acta 54: 2015-2050.

McLennan, S.; Bock, B.; Hemmin, S.; Hurowitz, J.; Lev, S.; McDaniel, D. 2003. The roles of provenance and sedimentary processes in the geochemistry of sedimentary rocks. In Geochemistry of sediments and sedimentary rocks: evolutionary considerations to mineral deposit-forming environments (Lentz, D.; editor). Geological association of Canada. Geotex 4: 7-38.

Murra, J.; Baldo, E.; Galindo, C.; Casquet, C.; Pankhurst, R.; Rapela, R.; Dahlquist, J.2011. Sr, C and O isotope composition of marbles from the Sierra deAncasti, Eastern Sierras Pampeanas, Argentina: age and constraints for the Neoproterozoic-lower Paleozoic evolution of the proto-Gondwana margin. Geologica Acta 9: 79-92.

Nakamura, N. 1974. Determination of REE, Ba, Mg, Na and $\mathrm{K}$ in carbonaceous and ordinary chondrites. Geochimica et Cosmochimica Acta 38: 757-773.

Otamendi, J.; Tibaldi, A.; Vujovich, G.; Viñao, G. 2008. Metamorphic evolution of migmatites from the deep Famatinian arc crust exposed in Sierras Valle Fértil-La Huerta, San Juan, Argentina. Journal of South American Earth Sciences 25: 313-335.

Otamendi, J.; Ducea, M.; Bergantz, G. 2012. Geological, petrological and geochemical evidence for progressive construction of an arc crustal section, Sierra de Valle Fértil, Famatinian arc, Argentina. Journal of Petrology 53 (4): 761-800.

Pankhurst, R.J.; Rapela, C.W. 1998. The Proto-Andean Margin of Gondwana: An Introduction. In The ProtoAndean Margin ofGondwana(Pankhurst, R.J.; Rapela, C.W.; editors). Geological Society of London Special Publication 142: 1-9.

Pankhurst, R.; Rapela, C.; Saavedra, J.; Baldo, E.; Dahlquist, J.; Pascua, I.; Fanning, C. 1998. The Famatinian magmatic arc in the central Sierras Pampeanas: an early to mid-Ordovician continental arc on the Gondwana margin. In The proto-Andean margin of Gondwana (Pankhurst, R.; Rapela, C.; editors). Geological Society of London, Special Publication 142: 343-397.

Pankhurst, R.; Rapela, C.; Fanning, C. 2000. Age and origin of coeval TTG, I- and S-type granites in the Famatinian belt of NW Argentina. Transactions of the Royal Society of Edinburgh, Earth Sciences 91: 151-168.

Pascua, I.; Saavedra, J.; Baldo, E. 1998. Metamorfismo y magmatismo del Paleozoico inferior en la zona central de las Sierras Pampeanas (SW de Gondwana). Geogaceta 20-7: 1672-1675.

Patocka, F.; Storch, P. 2004. Evolution of geochemistry and depositional setting of early Paleozoic siliciclastics of the Barrandian (Teplá-Barrandian unit, 
Bohemian massif, Czech Republic). International Journal of Earth Science 93 (5): 728-741.

Rapela,C.;Pankhurst,R.;Casquet,C.;Baldo,E.;Saavedra,J.; Galindo, C.; Fanning, C. 1998. The Pampean orogeny of the southern proto-Andes: Cambrian continental collision in the Sierras de Córdoba. In The protoAndean margin of Gondwana (Pankhurst, R.; Rapela, C.; editors). Geological Society London Special Publications 142: 181-217.

Rapela,C.;Pankhurst,R.;Casquet,C.; Fanning,C.; Baldo,E.; González-Casado, J.; Galindo, C.; Dahlquist, J. 2007. The Río de la Plata Craton and the assembly of SW Gondwana. Earth-Science Reviews 83: 49-82.

Siegesmund, S.; Steenken, A.; Martino, R.; Wemmer, K.; López de Luchi, M.; Frei, R.; Presnyakov, S.; Guereschi, A. 2010. Time constraints on the tectonic evolution of the Eastern Sierras Pampeanas (central Argentina). International Journal of Earth Science (Geologische Rundschau) 99: 1199-1226.

Sims, J.; Ireland, T.; Camacho, A.; Lyons, P.; Pieters, P.; Skirrow, R.; Stuart-Smith, P.; Miró, R. 1998. U-Pb, $\mathrm{Th}-\mathrm{Pb}$ and $\mathrm{Ar} / \mathrm{Ar}$ geochronology from the southern Sierras Pampeanas, Argentina: implications for the Palaeozoic tectonic development of the western Gondwana margin. In The proto-Andean margin of Gondwana (Pankhurst, R.; Rapela, C.; editors). Geological Society London, Special Publication 142: 259-281.

Steenken,A.; Siegesmund, S.; López de Luchi, M.; Frei, R.; Wemmer, K. 2006. Neoproterozoic to early Palaeozoic events in the Sierra de San Luis: implications for the Famatinian geodynamics in the Eastern Sierras
Pampeanas (Argentina). Journal of the Geological Society of London 163: 965-982.

Steenken, A.; López de Luchi, M.; Martínez Dopico, C.; Drobe, M.; Wemmer, K.; Siegesmund, S. 2011. The Neoproterozoic-early Paleozoic metamorphic and magmatic evolution of the Eastern Sierras Pampeanas: An Overview. In International Journal of Earth Sciences 100: 465-488.

Verdecchia, S.; Baldo, E. 2010. Geoquímica y procedencia de los metasedimentos orodvícicos del complejo metamórfico La Cébila, Provincia de La Rioja, Argentina. Revista Mexicana de Ciencias Geológicas 27 (1): 97-11.

Verdecchia, S.; Baldo, E.; Benedetto, J.; Borghi, P. 2007. The first shelly faunas from metamorphic rocks of the Sierras Pampeanas (La Cébila formation, Sierra de Ambato, Argentina): age and paleogeographic implications. Ameghiniana 44: 493-498.

Verdecchia, S.; Casquet, C.; Baldo, E.; Rapela, C.; Pankhurst, R.; Fanning, C.; Galindo, C. 2011. Mid to late Cambrian docking of the Río de la Plata Craton to southwestern Gondwana: age constraints from $\mathrm{U}-\mathrm{Pb}$ shrimp detrital zircon ages from Sierras de Ambato and Velasco (Sierras Pampeanas, Argentina). Journal of Geological Society of London 168: 1061-1071.

Zimmermann, U. 2005. Provenance studies of very lowto low-grade metasedimentary rocks of the Puncoviscana complex, northwest Argentina. In Terrane processes at the margins of Gondwana (Vaughan, A.; Leat, P.; Pankhurst, R.; editors). Geological Society London, Special Publication 246: 381-416. 
APÉNDICE, ANÁLISIS GEOQUíMICOS DE ROCAS METASEDIMENTARIAS DEL COMPLEJO METAMÓRFICO LOS LLANOS Y DEL COMPLEJO METAMÓRFICO LA CÉBILA.

\begin{tabular}{|c|c|c|c|c|c|c|c|c|c|c|c|c|c|c|c|}
\hline \multirow{4}{*}{$\begin{array}{l}\text { Litología } \\
\text { Latitud }\end{array}$} & \multicolumn{8}{|c|}{ Complejo Metamórfico Los Llanos } & \multicolumn{7}{|c|}{ Complejo Metamórfico La Cébila } \\
\hline & \multicolumn{3}{|c|}{ Metapsamitas } & \multicolumn{5}{|c|}{ Metapelitas } & \multicolumn{7}{|c|}{ Metapsamitas ( $\left.{ }^{2}\right)$} \\
\hline & \multirow{2}{*}{$\begin{array}{c}\text { Esquisto } \\
\text { cuarzoso con } \\
\text { andalucita (') } \\
30^{\circ} 18^{\prime} 17,00^{\prime \prime} \mathrm{S}\end{array}$} & \multirow{2}{*}{$\begin{array}{c}\begin{array}{c}\text { Corneana } \\
\text { cuarzosa }\end{array} \\
30^{\circ} 18^{\prime} 45,00 \text { 's }\end{array}$} & \multirow{2}{*}{$\begin{array}{l}\text { Metapsamita } \\
30^{\circ} 20^{\prime} 42,00 ” S\end{array}$} & \multirow{2}{*}{$\begin{array}{l}\text { Esquisto cuarzo } \\
\text { micáceo con } \\
\text { andalucita }\end{array}$} & $\begin{array}{l}\text { Esquisto cuarzo } \\
\text { micáceo con } \\
\text { andalucita }\end{array}$ & Corneana & Gneis & Filita & \multicolumn{3}{|c|}{ Metapsamitas de bajo grado metamórfico } & \multicolumn{2}{|c|}{ Metapsamitas cuarzo-micáceas } & \multicolumn{2}{|c|}{$\begin{array}{l}\text { Metapsamitas de bajo grado } \\
\text { metamórfico }\end{array}$} \\
\hline & & & & & $30^{\circ} 20^{\prime} 42,00^{\prime} \mathrm{S}$ & $30^{\circ} 20^{\prime} 39,00^{\prime} \mathrm{S}$ & $30^{\circ} 19^{\prime} 35,00^{\prime} \mathrm{S}$ & $30^{\circ} 33^{\prime} 6,00^{\prime}$ 's & $29^{\circ} 12^{\prime} 14,50 ” \mathrm{~S}$ & $29^{\circ} 11^{\prime} 49,70 ” \mathrm{~S}$ & $29^{\circ} 10^{\prime} 33,40 \prime \mathrm{S}$ & $28^{\circ} 43^{\prime} 15,40 ” S$ & $28^{\circ} 43^{\prime} 15,40 ” \mathrm{~S}$ & $28^{\circ} 49^{\prime} 23,50 ” \mathrm{~S}$ & $28^{\circ} 50^{\prime} 0,70^{\prime} \mathrm{S}$ \\
\hline Longitud & $66^{\circ} 32^{\prime} 19,00^{\prime \prime} \mathrm{W}$ & $66^{\circ} 32^{\prime} 33,00^{\prime} \mathrm{W}$ & $66^{\circ} 31^{\prime} 48,00^{\prime \prime} \mathrm{W}$ & $66^{\circ} 32^{\prime} 33,00^{\prime} \mathrm{W}$ & $66^{\circ} 31^{\prime} 48,00^{\prime} \mathrm{W}$ & $66^{\circ} 32^{\circ} 9,00^{\prime} \mathrm{W}$ & $66^{\circ} 32^{\prime} 10,00^{\prime} \mathrm{W}$ & $66^{\circ} 21^{\prime}, 6,00^{\prime} \mathrm{W}$ & $66^{\circ} 46^{\prime} 26,40^{\prime \prime} \mathrm{W}$ & $66^{\circ} 47^{\prime}, 2,40 ” \mathrm{~W}$ & $66^{\circ} 48^{\prime} 15,80^{\prime} \mathrm{W}$ & $66^{\circ} 21^{\prime} 42,20^{\prime \prime} \mathrm{W}$ & $66^{\circ} 21^{\prime} 42,20^{\prime} \mathrm{W}$ & $66^{\circ} 24^{\prime} 17,20^{\prime} \mathrm{W}$ & $66^{\circ} 24^{\prime} 10,10^{\prime} \mathrm{W}$ \\
\hline Muestra & GAR-33 & GAR-38 & DIF-56 & GAR-36 & DIF-57 & DIF-61 & ESP-70 & SAB-144 & CAN-304 & CAN-306 & CAN-318 & CEB-353 & CEB-354 & CEB-386 & CEB-388 \\
\hline \multicolumn{16}{|c|}{ (\% en peso) } \\
\hline $\mathrm{SiO}_{2}$ & 84,55 & 87,89 & 85,19 & 58,87 & 55,4 & 60 & 62,22 & 61,44 & 87,69 & 86,88 & 82,18 & 76,93 & 76,09 & 74,82 & 73,15 \\
\hline $\mathrm{TiO}_{2}$ & 0,32 & 0,42 & 0,45 & 0,91 & 1,07 & 0,83 & 0,90 & 0,84 & 0,12 & 0,14 & 0,22 & 0,15 & 0,27 & $\mathbf{0 , 0 7}$ & 0,28 \\
\hline $\mathrm{Al}_{2} \mathrm{O}_{3}$ & 6,86 & 5,76 & 5,94 & 18,43 & 21,67 & 20,07 & 19,05 & 19,32 & 6,74 & 6,13 & 7,38 & 5,73 & 12,06 & 11,92 & 11,75 \\
\hline $\mathrm{Fe}_{2} \mathrm{O}_{3}$ & 1,57 & 1,8 & 2,76 & 7,25 & 8,19 & 7,98 & 7,68 & 6,76 & 1,56 & 1,15 & 2,49 & 8,28 & 3,43 & 1,62 & 4,13 \\
\hline $\mathrm{MnO}$ & 0,02 & 0,03 & 0,02 & 0,10 & 0,08 & 0,08 & 0,20 & 0,06 & 0,02 & 0,03 & 0,04 & 0,06 & 0,03 & 0,02 & 0,04 \\
\hline $\mathrm{MgO}$ & 0,43 & 0,50 & 1,01 & 2,96 & 3,23 & 2,94 & 3,04 & 2,38 & 0,18 & 0,41 & 0,86 & 1,72 & 0,59 & 0,68 & 1,67 \\
\hline $\mathrm{CaO}$ & 0,29 & 0,45 & 0,85 & 0,25 & 0,64 & 0,22 & 0,34 & 0,35 & 0,04 & 0,83 & 0,82 & 0,14 & 0,46 & 1,94 & 0,87 \\
\hline $\mathrm{Na}_{0} 0$ & 1,15 & 1,21 & 1,39 & 0,46 & 1,90 & 0,83 & 1,34 & 1,31 & 0,65 & 2,21 & 2,98 & 0,63 & 0,84 & 2,00 & 0,40 \\
\hline $\mathrm{K}, \mathrm{O}$ & 2,4 & 1,13 & 0,97 & 4,49 & 5,33 & 5,34 & 4,80 & 4,93 & 0,44 & 0,40 & 0,25 & 0,92 & 3,24 & 1,50 & 2,76 \\
\hline $\mathrm{P}_{2} \mathrm{O}_{5}$ & 0,08 & 0,06 & 0,12 & 0,13 & 0,11 & 0,14 & 0,18 & 0,19 & 0,05 & 0,10 & 0,13 & 0,08 & 0,27 & 0,10 & 0,25 \\
\hline LOI & 0,57 & 0,59 & 0,36 & 4,89 & 2,00 & 1,56 & 0,86 & 3,15 & 2,59 & 1,00 & 1,59 & 2,69 & 1,52 & 2,87 & 3,54 \\
\hline total & 98,24 & 99,84 & 99,05 & 98,72 & 99,61 & 99,99 & 100,59 & 100,72 & 100,07 & 99,27 & 98,94 & 97,32 & 98,79 & 97,53 & 98,83 \\
\hline \multicolumn{16}{|l|}{ (ppm) } \\
\hline $\mathrm{Cs}$ & 2,6 & 2,7 & 4,7 & 11,4 & 12,6 & 25,5 & 12,7 & 14,9 & 3,4 & 1,6 & 0,7 & 13,1 & 11,4 & 8,9 & 4,8 \\
\hline $\mathrm{Rb}\left(^{(3)}\right.$ & 92 & 59,6 & 56 & 205,8 & 218,8 & 241,1 & 181,3 & 214,8 & 26,3 & 17,6 & 10,8 & 111,1 & 141,5 & 84,1 & 125,9 \\
\hline $\operatorname{Sr}\left(^{(3)}\right.$ & 62 & 54 & 148 & 59 & 164 & 64 & 71 & 74 & 253,8 & 277,1 & 381,4 & 7,5 & 293,7 & 171,9 & 96 \\
\hline $\mathrm{Ba}\left({ }^{3}\right)$ & 302 & 152 & 128 & 455 & 747 & 667 & 543 & 715 & 210,5 & 134,8 & 201,5 & 35,2 & 859,1 & 405,2 & 711,9 \\
\hline $\mathrm{La}$ & 27,4 & 38,1 & 27 & 58,5 & 66 & 53,7 & 55,1 & 31,8 & 35,9 & 20 & 33,3 & 14,2 & 35,4 & 31,2 & 34,8 \\
\hline $\mathrm{Ce}$ & 63,4 & 87 & 63,3 & 124,5 & 136,3 & 111,7 & 120,7 & 69,7 & 75,7 & 45,8 & 67,2 & 31,4 & 73,4 & 63,9 & 73,9 \\
\hline $\operatorname{Pr}$ & 5,1 & 6,8 & 5,7 & 10,9 & 11,8 & 9,9 & 10,1 & 6,1 & 8,8 & 5,2 & 7,9 & 3,8 & 8,8 & 7,6 & 8,9 \\
\hline $\mathrm{Nd}$ & 22,2 & 29,2 & 26,2 & 46,5 & 51,3 & 42,5 & 44,2 & 25,2 & 32,5 & 20,1 & 30 & 15,9 & 33,6 & 28,6 & 34,7 \\
\hline $\mathrm{Sm}$ & 4,8 & 5,8 & 6,3 & 10 & 10,5 & 9,1 & 8,9 & 5 & 6,1 & 4,4 & 6,1 & 4,1 & 7,4 & 6,1 & 8 \\
\hline $\mathrm{Eu}$ & 0,9 & 1 & 1,2 & 1,7 & 2,2 & 1,6 & 1,7 & 1 & 1,2 & 0,9 & 1,1 & 0,3 & 1,6 & 1,3 & 1,6 \\
\hline $\mathrm{Gd}$ & 4,2 & 5 & 5,8 & 8 & 9 & 7,7 & 7,7 & 4,4 & 4,5 & 3,9 & 5,4 & 4,6 & 6,9 & 4,9 & 7,9 \\
\hline $\mathrm{Tb}$ & 0,7 & 0,9 & 1 & 1,4 & 1,5 & 1,3 & 1,3 & 0,8 & 0,6 & 0,6 & 0,8 & 0,7 & 1,1 & 0,6 & 1,3 \\
\hline Dy & 4,1 & 4,6 & 5,6 & 7,7 & 8,2 & 7,1 & 6,8 & 5,1 & 2,6 & 3,8 & 4,7 & 4,4 & 6,6 & 3,4 & 7,8 \\
\hline Но & 0,8 & 0,9 & 1,1 & 1,5 & 1,6 & 1,4 & 1,3 & 1,1 & 0,5 & 0,8 & 0,9 & 0,8 & 1,3 & 0,6 & 1,5 \\
\hline $\mathrm{Er}$ & 2,5 & 2,7 & 3,2 & 4,5 & 4,9 & 4,1 & 3,9 & 3,6 & 1,2 & 2,1 & 2,5 & 2,1 & 3,4 & 1,6 & 4 \\
\hline $\mathrm{Tm}$ & 0,4 & 0,4 & 0,5 & 0,7 & 0,8 & 0,7 & 0,6 & 0,7 & 0,2 & 0,3 & 0,4 & 0,3 & 0,5 & 0,2 & 0,6 \\
\hline $\mathrm{Yb}$ & 2,4 & 2,7 & 2,8 & 3,9 & 4,3 & 3,6 & 3,5 & 3,7 & 1,1 & 2 & 2,2 & 1,8 & 3 & 1,4 & 3,5 \\
\hline $\mathrm{Lu}$ & 0,4 & 0,4 & 0,4 & 0,6 & 0,7 & 0,6 & 0,5 & 0,6 & 0,2 & 0,3 & 0,3 & 0,3 & 0,5 & 0,2 & 0,5 \\
\hline $\mathrm{U}$ & 3,1 & 3,7 & 2,5 & 4,6 & 4,6 & 4,2 & 4,2 & 4,6 & 1,4 & 2,4 & 2,5 & 7,8 & 3,7 & 2,7 & 6,1 \\
\hline Th & 13,6 & 15,1 & 11,6 & 21,3 & 23,7 & 20,6 & 9,7 & 19,8 & 7,9 & 7,3 & 11 & 5,8 & 12,8 & 11,4 & 12,9 \\
\hline$Y\left({ }^{3}\right)$ & 21 & 25 & 30 & 38 & 42 & 36 & 34 & 28 & 11 & 20,5 & 23,9 & 20,7 & 35,7 & 16,5 & 42,4 \\
\hline $\mathrm{Nb}\left({ }^{3}\right)$ & 9 & 10 & 10 & 20 & 22 & 18 & 19 & 18 & 5,5 & 6,1 & 9,4 & 7,3 & 11,7 & 9,9 & 12,9 \\
\hline $\operatorname{Zr}\left({ }^{(3)}\right.$ & 265 & 463 & 320 & 167 & 195 & 139 & 187 & 167 & 300,2 & 429,2 & 408,1 & 181,7 & 219,2 & 107 & 272,1 \\
\hline $\mathrm{Hf}$ & 7,9 & 12,4 & 9,8 & 5,4 & 6,1 & 4,4 & 5,4 & 5,3 & 7,9 & 11 & 10,7 & 4,9 & 6 & 3,5 & 7,4 \\
\hline $\mathrm{Sc}$ & 4,4 & 4,5 & 5,2 & 18,7 & 21,5 & 19,3 & 16,2 & 16,9 & 2,2 & 3,3 & 5,1 & 7,7 & 10,5 & 4,7 & 11,1 \\
\hline
\end{tabular}




\begin{tabular}{|c|c|c|c|c|c|c|c|c|c|c|c|c|c|c|c|}
\hline \multirow{4}{*}{$\begin{array}{l}\text { Litología } \\
\text { Latitud }\end{array}$} & \multicolumn{8}{|c|}{ Complejo Metamórfico Los Llanos } & \multicolumn{7}{|c|}{ Complejo Metamórfico La Cébila } \\
\hline & \multicolumn{3}{|c|}{ Metapsamitas } & \multicolumn{5}{|c|}{ Metapelitas } & \multicolumn{7}{|c|}{ Metapsamitas $\left({ }^{(}\right)$} \\
\hline & \multirow{2}{*}{$\begin{array}{c}\text { Esquisto } \\
\text { cuarzoso con } \\
\text { andalucita (') } \\
30^{\circ} 18^{\prime} 17,00 " S\end{array}$} & \multirow{2}{*}{$\begin{array}{c}\begin{array}{c}\text { Corneana } \\
\text { cuarzosa }\end{array} \\
30^{\circ} 18^{\prime} 45,00^{\prime \prime} \mathrm{S}\end{array}$} & \multirow{2}{*}{$\begin{array}{l}\text { Metapsamita } \\
30^{\circ} 20^{\prime} 42,00^{\prime \prime} \mathrm{S}\end{array}$} & \multirow{2}{*}{$\begin{array}{c}\text { Esquisto } \\
\text { cuarzo micáceo } \\
\text { con andalucita } \\
30^{\circ} 18^{\prime} 45,00 ” S\end{array}$} & \multirow{2}{*}{$\begin{array}{c}\text { Esquisto } \\
\text { micáceo con } \\
\text { andalucita } \\
30^{\circ} 20^{\prime} 42,00 \text { 's }\end{array}$} & \multirow{2}{*}{$\begin{array}{c}\text { Corneana } \\
30^{\circ} 20^{\prime} 39,00^{\prime \prime S}\end{array}$} & \multirow{2}{*}{$\begin{array}{c}\text { Gneis } \\
30^{\circ} 19^{\prime} 35,00^{\prime} \mathrm{S}\end{array}$} & \multirow{2}{*}{$\begin{array}{c}\text { Filita } \\
30^{\circ} 33^{\prime} 6,00 " \mathrm{~S}\end{array}$} & \multicolumn{3}{|c|}{ Metapsamitas de bajo grado metamórfico } & \multicolumn{2}{|c|}{ Metapsamitas cuarzo-micáceas } & \multicolumn{2}{|c|}{$\begin{array}{l}\text { Metapsamitas de bajo grado } \\
\text { metamórfico }\end{array}$} \\
\hline & & & & & & & & & $29^{\circ} 12^{\prime} 14,50^{\prime} \mathrm{S}$ & $29^{\circ} 11^{\prime} 49,70 ” \mathrm{~S}$ & $29^{\circ} 10^{\prime} 33,40^{\prime} \mathrm{S}$ & $28^{\circ} 43^{\prime} 15,40 ” \mathrm{~S}$ & $28^{\circ} 43^{\prime} 15,40 ” S$ & $28^{\circ} 49^{\prime} 23,50 ” \mathrm{~S}$ & $28^{\circ} 50^{\prime} 0,70^{\prime \prime S}$ \\
\hline Longitud & $66^{\circ} 32^{\prime} 19,00^{\prime \prime} \mathrm{W}$ & $66^{\circ} 32 ’ 33,00 ” \mathrm{~W}$ & $66^{\circ} 31^{\prime} 48,00^{\prime \prime} \mathrm{W}$ & $66^{\circ} 32 ’ 33,00^{\prime \prime} \mathrm{W}$ & $66^{\circ} 31^{\prime} 48,00^{\prime \prime} \mathrm{W}$ & $66^{\circ} 32^{\prime} 9,00^{\prime \prime W}$ & $66^{\circ} 32^{\prime} 10,00^{\prime \prime} \mathrm{W}$ & $66^{\circ} 21^{\prime} 6,00^{\prime \prime} \mathrm{W}$ & $66^{\circ} 46^{\prime 2} 26,40 ” \mathrm{~W}$ & $66^{\circ} 47^{\prime 2} 2,40^{\prime \prime W}$ & $66^{\circ} 48^{\prime} 15,80^{\prime \prime} \mathrm{W}$ & $66^{\circ} 21^{\prime} 42,20^{\prime \prime} \mathrm{W}$ & $66^{\circ} 21^{\prime} 42,20^{\prime \prime} \mathrm{W}$ & $66^{\circ} 24^{\prime} 17,20^{\prime \prime} \mathrm{W}$ & $66^{\circ} 24^{\prime} 10,10^{\prime \prime W}$ \\
\hline Muestra & GAR-33 & GAR-38 & DIF-56 & GAR-36 & DIF-57 & DIF-61 & ESP-70 & SAB-144 & CAN-304 & CAN-306 & CAN-318 & CEB-353 & CEB-354 & CEB-386 & CEB-388 \\
\hline $\mathrm{Ni}\left({ }^{3}\right)$ & 4 & 9 & 8 & 41 & 39 & 48 & 50 & 46 & - & - & - & - & - & - & - \\
\hline $\operatorname{Cr}\left({ }^{3}\right)$ & 60,6 & 31,3 & 58,1 & 80,6 & 103 & 95,4 & 74,3 & 80,8 & - & - & - & - & - & - & - \\
\hline $\mathrm{Cu}$ & 3 & 9 & 5 & 30 & 16 & 39 & 18 & 23 & - & - & - & - & - & - & - \\
\hline s & $<50$ & $<50$ & 380 & 260 & 760 & 1860 & 80 & $<50$ & - & - & - & - & - & - & - \\
\hline $\mathrm{Pb}\left({ }^{3}\right)$ & 23 & 14 & 19 & 25 & 28 & 23 & 27 & 21 & 26,8 & 15,1 & 38 & 2,5 & 12,6 & 24,5 & 12,7 \\
\hline $\mathrm{Zn}\left({ }^{3}\right)$ & 25 & 26 & 33 & 122 & 75 & 131 & 120 & 111 & - & - & - & - & - & - & - \\
\hline $\mathrm{V}\left({ }^{3}\right)$ & 36 & 36 & 32 & 185 & 145 & 130 & 106 & 120 & - & - & - & - & - & - & - \\
\hline $\mathrm{Al}_{2} \mathrm{O}_{3} / \mathrm{K}_{2} \mathrm{O}$ & 2,86 & 5,1 & 6,12 & 4,1 & 4,07 & 3,76 & 3,97 & 3,92 & 15,32 & 15,33 & 29,52 & 6,23 & 3,72 & 7,95 & 4,26 \\
\hline $\mathrm{Rb} / \mathrm{Sr}$ & 1,48 & 1,1 & 0,38 & 3,49 & 1,33 & 3,77 & 2,55 & 2,9 & 0,1 & 0,06 & 0,03 & 14,89 & 0,48 & 0,49 & 1,31 \\
\hline $\left.\mathrm{Eu} / \mathrm{Eu}^{*}{ }^{4}\right)$ & 0,62 & 0,56 & 0,61 & 0,59 & 0,7 & 0,61 & 0,64 & 0,67 & 0,72 & 0,67 & 0,62 & 0,22 & 0,68 & 0,71 & 0,6 \\
\hline $\begin{array}{l}\mathrm{Mg} \#(\mathrm{Mg} / \\
(\mathrm{Mg}+\mathrm{Fe}))\end{array}$ & 0,35 & 0,35 & 0,42 & 0,45 & 0,44 & 0,42 & 0,44 & 0,41 & 0,19 & 0,41 & 0,41 & 0,29 & 0,25 & 0,45 & 0,44 \\
\hline $\mathrm{Cr} / \mathrm{Th}$ & 4,5 & 2,1 & 5,0 & 3,8 & 4,3 & 4,6 & 7,7 & 4,1 & - & - & - & - & - & - & - \\
\hline $\mathrm{V} / \mathrm{Ni}$ & 9,0 & 4,0 & 4,0 & 4,5 & 3,7 & 2,7 & 2,1 & 2,6 & - & - & - & - & - & - & - \\
\hline $\mathrm{Sc} /(\mathrm{Sc}+\mathrm{Th}+\mathrm{Zr} / 10)$ & 0,1 & 0,07 & 0,11 & 0,33 & 0,33 & 0,36 & 0,36 & 0,32 & 0,05 & 0,06 & 0,09 & 0,24 & 0,23 & 0,18 & 0,22 \\
\hline $\mathrm{Th} /(\mathrm{Sc}+\mathrm{Th}+\mathrm{Zr} / 10)$ & 0,31 & 0,23 & 0,24 & 0,38 & 0,37 & 0,38 & 0,22 & 0,37 & 0,2 & 0,14 & 0,19 & 0,18 & 0,28 & 0,42 & 0,25 \\
\hline $\begin{array}{l}(\mathrm{Zr} / 10) / \\
(\mathrm{Sc}+\mathrm{Th}+\mathrm{Zr} / 10)\end{array}$ & 0,6 & 0,7 & 0,66 & 0,29 & 0,3 & 0,26 & 0,42 & 0,31 & 0,75 & 0,8 & 0,72 & 0,57 & 0,48 & 0,4 & 0,53 \\
\hline $\mathrm{Th} /(\mathrm{Th}+\mathrm{La}+\mathrm{Sc})$ & 0,3 & 0,26 & 0,26 & 0,22 & 0,21 & 0,22 & 0,12 & 0,29 & 0,17 & 0,24 & 0,22 & 0,21 & 0,22 & 0,24 & 0,22 \\
\hline $\mathrm{La} /(\mathrm{Th}+\mathrm{La}+\mathrm{Sc})$ & 0,6 & 0,66 & 0,62 & 0,59 & 0,59 & 0,57 & 0,68 & 0,46 & 0,78 & 0,65 & 0,67 & 0,51 & 0,6 & 0,66 & 0,59 \\
\hline $\mathrm{Sc} /(\mathrm{Th}+\mathrm{La}+\mathrm{Sc})$ & 0,1 & 0,08 & 0,12 & 0,19 & 0,19 & 0,21 & 0,2 & 0,25 & 0,05 & 0,11 & 0,1 & 0,28 & 0,18 & 0,1 & 0,19 \\
\hline $\mathrm{La} / \mathrm{Th}$ & 2 & 2,5 & 2,3 & 2,7 & 2,8 & 2,6 & 5,7 & 1,6 & 4,5 & 2,8 & 3 & 2,4 & 2,8 & 2,7 & 2,7 \\
\hline $\mathrm{Ti} / \mathrm{Zr}$ & 7,2 & 5,4 & 8,4 & 32,7 & 32,9 & 35,8 & 28,9 & 30,2 & 2,4 & 2 & 3,2 & 4,9 & 7,4 & 3,9 & 6,2 \\
\hline $\mathrm{La} / \mathrm{Sc}$ & 6,2 & 8,5 & 5,2 & 3,1 & 3,1 & 2,8 & 3,4 & 1,9 & 16,4 & 6,1 & 6,6 & 1,9 & 3,4 & 6,6 & 3,1 \\
\hline $\mathrm{Th} / \mathrm{Sc}$ & 3,1 & 3,4 & 2,2 & 1,1 & 1,1 & 1,1 & 0,6 & 1,2 & 3,6 & 2,2 & 2,2 & 0,8 & 1,2 & 2,4 & 1,2 \\
\hline $\mathrm{Zr} / \mathrm{Sc}$ & 60,2 & 102,9 & 61,5 & 8,9 & 9,1 & 7,2 & 11,5 & 9,9 & 136,8 & 130,8 & 80,5 & 23,6 & 20,9 & 22,6 & 24,5 \\
\hline $\operatorname{CIA}\left({ }^{5}\right)$ & 59 & 60 & 56 & 76 & 69 & 74 & 72 & 72 & - & - & - & - & - & - & - \\
\hline
\end{tabular}

Normalización a la composición del Condrito $(9$

\begin{tabular}{llll|rrrr} 
N-La & 83 & 116 & 82 & 178 & 201 & 163 & 167 \\
N-Ce & 73 & 101 & 73 & 144 & 158 & 129 & 139 \\
N-Pr & 42 & 56 & 47 & 89 & 97 & 81 & 83 \\
N-Nd & 35 & 46 & 42 & 74 & 81 & 67 & 70 \\
N-Sm & 24 & 29 & 31 & 49 & 52 & 45 & 44 \\
N-Eu & 12 & 13 & 15 & 22 & 29 & 21 & 22 \\
N-Gd & 15 & 18 & 21 & 29 & 33 & 28 & 28 \\
N-Tb & 15 & 18 & 22 & 29 & 31 & 28 & 26 \\
N-Dy & 12 & 13 & 16 & 22 & 24 & 21 & 20 \\
N-Ho & 11 & 13 & 15 & 21 & 23 & 19 & 18
\end{tabular}

\begin{tabular}{l|r}
97 & 109 \\
81 & 87 \\
50 & 72 \\
40 & 52 \\
24 & 30 \\
13 & 16 \\
16 & 16 \\
17 & 12 \\
15 & 8 \\
16 & 6
\end{tabular}


continuación apéndice.

\begin{tabular}{|c|c|c|c|c|c|c|c|c|c|c|c|c|c|c|c|}
\hline \multirow{4}{*}{$\begin{array}{l}\text { Litología } \\
\text { Latitud }\end{array}$} & \multicolumn{8}{|c|}{ Complejo Metamórfico Los Llanos } & \multicolumn{7}{|c|}{ Complejo Metamórfico La Cébila } \\
\hline & \multicolumn{3}{|c|}{ Metapsamitas } & \multicolumn{5}{|c|}{ Metapelitas } & \multicolumn{7}{|c|}{ Metapsamitas $\left({ }^{2}\right)$} \\
\hline & \multirow{2}{*}{$\begin{array}{c}\text { Esquisto } \\
\text { cuarzoso con } \\
\text { andalucita (') } \\
30^{\circ} 18^{\prime} 17,00^{\prime} \mathrm{S}\end{array}$} & \multirow{2}{*}{$\begin{array}{c}\begin{array}{c}\text { Corneana } \\
\text { cuarzosa }\end{array} \\
30^{\circ} 18^{\prime} 45,00^{\prime} \text { S }\end{array}$} & \multirow{2}{*}{$\begin{array}{l}\text { Metapsamita } \\
30^{\circ} 20^{\prime} 42,00^{\prime} \text { S }\end{array}$} & \multirow{2}{*}{$\begin{array}{c}\text { Esquisto cuarzo } \\
\text { micáceo con } \\
\text { andalucita } \\
\\
30^{\circ} 18^{\prime} 45,00 " S\end{array}$} & \multirow{2}{*}{$\begin{array}{c}\text { Esquisto cuarzo } \\
\text { micáceo con } \\
\text { andalucita } \\
\\
3^{\circ} 0^{\prime} 0^{\prime} 42,00 \text { "S }\end{array}$} & \multirow{2}{*}{$\begin{array}{c}\text { Corneana } \\
30^{\circ} 20^{\prime} 39,00^{\prime} \mathrm{S}\end{array}$} & \multirow{2}{*}{$\begin{array}{c}\text { Gneis } \\
30^{\circ} 19^{\prime} 35,00 ” S\end{array}$} & \multirow{2}{*}{$\begin{array}{c}\text { Filita } \\
\text { 30'33'6,00”S }\end{array}$} & \multicolumn{3}{|c|}{ Metapsamitas de bajo grado metamórfico } & \multicolumn{2}{|c|}{ Metapsamitas cuarzo-micáceas } & \multicolumn{2}{|c|}{$\begin{array}{l}\text { Metapsamitas de bajo grado } \\
\text { metamórfico }\end{array}$} \\
\hline & & & & & & & & & $29^{\circ} 12^{\prime} 14,50^{\prime} \mathrm{S}$ & $29^{\circ} 11^{\prime} 49,70 ” \mathrm{~S}$ & $29^{\circ} 10^{\prime} 33,40 ” \mathrm{~S}$ & $28^{\circ} 43^{\prime} 15,40 ” S$ & $28^{\circ} 43^{\prime} 15,40 ” S$ & $28^{\circ} 49^{\prime} 23,50 ” \mathrm{~S}$ & $28^{\circ} 50^{\prime} 0,70^{\prime \prime S}$ \\
\hline Longitud & $66^{\circ} 32^{\prime} 19,00^{\prime \prime} \mathrm{W}$ & $66^{\circ} 32^{\prime} 33,00^{\prime \prime} \mathrm{W}$ & $66^{\circ} 31^{\prime} 48,00^{\prime \prime W}$ & $66^{\circ} 32^{\prime} 33,00^{\prime \prime W}$ & $66^{\circ} 31^{\prime} 48,00^{\prime \prime} \mathrm{W}$ & $66^{\circ} 32^{\prime} 9,00^{\prime \prime W}$ & $66^{\circ} 32^{\prime} 10,00^{\prime \prime} \mathrm{W}$ & $66^{\circ} 21^{\prime} 6,00^{\prime \prime} \mathrm{W}$ & $66^{\circ} 46^{\prime 2} 26,40^{\prime \prime} \mathrm{W}$ & $66^{\circ} 47^{\prime 2,40 ” W}$ & $66^{\circ} 48^{\prime} 15,80^{\prime \prime W}$ & $66^{\circ} 21^{\prime} 42,20^{\prime \prime} \mathrm{W}$ & $66^{\circ} 21^{\prime} 42,20^{\prime \prime} \mathrm{W}$ & $66^{\circ} 24^{\prime} 17,20^{\prime \prime W}$ & $66^{\circ} 24^{\prime} 10,10^{\prime \prime} \mathrm{W}$ \\
\hline Muestra & GAR-33 & GAR-38 & DIF-56 & GAR-36 & DIF-57 & DIF-61 & ESP-70 & SAB-144 & CAN-304 & CAN-306 & CAN-318 & CEB-353 & CEB-354 & СЕB-386 & CEB-388 \\
\hline N-Er & 11 & 12 & 14 & 20 & 22 & 18 & 17 & 16 & 5 & 9 & 11 & 9 & 15 & 7 & 18 \\
\hline $\mathrm{N}-\mathrm{Tm}$ & 13 & 14 & 15 & 22 & 23 & 20 & 19 & 20 & 5 & 10 & 11 & 9 & 15 & 7 & 18 \\
\hline $\mathrm{N}-\mathrm{Yb}$ & 11 & 12 & 13 & 18 & 19 & 16 & 16 & 17 & 5 & 9 & 10 & 8 & 13 & 6 & 16 \\
\hline N-Lu & 11 & 12 & 13 & 18 & 20 & 17 & 16 & 17 & 5 & 10 & 10 & 8 & 14 & 6 & 16 \\
\hline
\end{tabular}

Notas: Todo el Fe está expresado como $\mathrm{Fe}_{2} \mathrm{O}_{3}$.

$\mathrm{Mg} \#=\mathrm{MgO} /(\mathrm{MgO}+\mathrm{FeO})$. Los óxidos están expresados en fracción molar.

Los elementos Ga, Sn, Mo, Ag, Au, As, Be, Bi, Cd, Hg, In, Ir, Se, Br, Tl, Ge, Sb de las muestras de Los Llanos fueron analizados, pero no incluidos en la presente tabla por ser muy bajos, inferiores o próximos al límite de detección.

(1) Muestra extraída de Dahlquist et al. (2005).

(2) Las concentraciones de elementos Mayores y La, Th y Hf corresponden a los publicados por Verdecchia y Baldo (2010).

(3) En las muestras del Complejo Metamórfico La Cébila, estos elementos fueron descritos en Verdecchia y Baldo (2010) a partir de análisis realizados mediante la Fluorescencia de Rayos X.

(4) $\mathrm{Eu} / \mathrm{Eu}^{*}$ calculado utilizando la composición del Condrito propuesta por Nakamura (1974).

(5) $\mathrm{CIA}$ (Índice de alteración química o Chemical Index of Alteration $)=\mathrm{Al}_{2} \mathrm{O}_{3} /\left(\mathrm{Al}_{2} \mathrm{O}_{3}+\mathrm{CaO}^{*}+\mathrm{Na}_{2} \mathrm{O}+\mathrm{K}_{2} \mathrm{O}\right.$. El CaO* representa únicamente el perteneciente a los silicatos y corregido según la sugerencia de McLennan $(1993)$.

(๑) Normalización realizada utilizando la composición del Condrito propuesta por Nakamura (1974) y completado con análisis de Pr, Tb, Ho, Tm de Boynton (1984). 